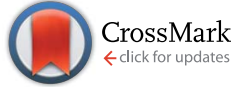

Cite this: Soft Matter, 2015, 11, 1708

Received 7th November 2014 Accepted 16th December 2014

DOI: $10.1039 / \mathrm{c} 4 \mathrm{sm} 02474 \mathrm{e}$

www.rsc.org/softmatter

\title{
Dynamics of high-speed micro-drop impact: numerical simulations and experiments at frame- to-frame times below 100 ns $\dagger$
}

\author{
Claas Willem Visser, ${ }^{* a}$ Philipp Erhard Frommhold, ${ }^{\text {b }}$ Sander Wildeman, ${ }^{\text {R }}$ Robert Mettin, ${ }^{\text {b }}$ \\ Detlef Lohse*a and Chao Sun*a
}

Technologies including (3D-) (bio-)printing, diesel engines, laser-induced forward transfer, and spray cleaning require optimization and therefore understanding of micrometer-sized droplets impacting at velocities beyond $10 \mathrm{~m} \mathrm{~s}^{-1}$. However, as yet, this regime has hardly been addressed. Here we present the first time-resolved experimental investigation of microdroplet impact at velocities up to $V_{0}=50 \mathrm{~m} \mathrm{~s}^{-1}$, on hydrophilic and -phobic surfaces at frame rates exceeding $10^{7}$ frames per second. A novel method to determine the $3 \mathrm{D}$-droplet profile at sub-micron resolution at the same frame rates is presented, using the fringe pattern observed from a bottom view. A numerical model, which is validated by the side- and bottom-view measurements, is employed to study the viscous boundary layer inside the droplet and the development of the rim. The spreading dynamics, the maximal spreading diameter, the boundary layer thickness, the rim formation, and the air bubble entrainment are compared to theory and previous experiments. In general, the impact dynamics are equal to millimeter-sized droplet impact for equal Reynolds-, Weber- and Stokes numbers (Re, We, and St, respectively). Using our numerical model, effective scaling laws for the progression of the boundary layer thickness and the rim diameter are provided. The dimensionless boundary layer thickness develops in time $(t)$ according to $\delta_{\mathrm{BL}} \sim D_{0} / \sqrt{\operatorname{Re}}(t / \tau)^{0.45}$, and the diameter of the rim develops as $D_{\operatorname{Rim}} \sim D_{0} / \sqrt{\mathrm{We}}(t / \tau)^{0.68}$, with drop diameter $D_{0}$ and inertial time scale $\tau=D_{0} / V_{0}$. These scalings differ from previously assumed, but never validated, values. Finally, no splash is observed, at variance with many predictions but in agreement with models including the influence of the surrounding gas. This confirms that the ambient gas properties are key ingredients for splash threshold predictions.

\section{Introduction}

Fast impact of small-scale droplets is a key phenomenon in applications such as diesel engines, ${ }^{\mathbf{1 , 2}}$ thermal spraying, ${ }^{3}$ spray cleaning, ${ }^{4}$ and novel manufacturing technologies based on inkjet printing technology ${ }^{5-7}$ or laser-induced forward transfer. ${ }^{8}$ However, the impact of these droplets is often poorly controlled, as it has hardly been visualized. The associated industrial problems include (but are not limited to) defects in metal electronics printing, ${ }^{3,9}$ reduced cell survival in spray treatment of burn wounds, ${ }^{\mathbf{1 0}}$ damage on semiconductor wafers, ${ }^{\mathbf{1 1}}$ and limited steel strip surface quality. ${ }^{12}$ To achieve a better understanding and thereby allow for improved process control, in this

\footnotetext{
${ }^{a}$ Physics of Fluids Group, Faculty of Science and Technology, J. M. Burgers Centre for Fluid Dynamics, University of Twente, The Netherlands. E-mail: c.visser@utwente.nl; d.lohse@utwente.nl; c.sun@utwente.nl

${ }^{b}$ Christian Doppler Laboratory for Cavitation and Micro-Erosion, Drittes Physikalisches Institut, Georg-August-University Göttingen, Göttingen, Germany

$\uparrow$ Electronic supplementary information (ESI) available. See DOI: $10.1039 / \mathrm{c} 4 \mathrm{sm} 02474 \mathrm{e}$
}

article we study the impact of fast, micro-scale droplets on a dry, smooth surface.

This is a challenging task, since for a droplet with diameter $D_{0}=50 \mu \mathrm{m}$ impacting at $50 \mathrm{~m} \mathrm{~s}^{-1}$, the impact time scale is $\tau=D_{0} / V_{0} \sim 1 \mu \mathrm{s}$. As this is comparable to the shutter time of high-quality fast cameras, these are still too slow to resolve the details of impact. Much shorter exposure times can be achieved using flash photography. ${ }^{13}$ However, with flash photography, only one or two frames per impacting droplet can be captured. Therefore, to obtain multiple frames of the impact using the flash approach, multiple droplet impacts with excellent reproducibility are required. So far, sufficiently reproducible droplets could only be made up to $20 \mathrm{~m} \mathrm{~s}^{-1} \cdot{ }^{14-16}$ As a result, singledroplet impact at high velocity $\left(V_{0}>10 \mathrm{~m} \mathrm{~s}^{-1}\right)$ and small diameters $\left(D_{0}<100 \mu \mathrm{m}\right)$ has hardly been visualized in detail, despite the vast interest for this subject in the context of the technologies mentioned.

In contrast, major progress has recently been made for $\mathrm{mm}$ sized droplet impact (for background see reviews ref. 34 and 35). We first provide a short overview of the these findings, as they provide the required context for our results on micro-droplets. 
As schematically shown in Fig. 1a, the impacting droplet spreads into a thin lamella until a maximum diameter is reached. The spreading of droplets was shown to be limited by either surface tension or viscosity. ${ }^{21}$ The formation of a viscous boundary layer was shown to be crucial, ${ }^{26,36,37}$ and has been included in several experimentally validated models. A selfsimilar profile of the droplet during spreading was theoretically predicted $^{36}$ and recently validated by $3 \mathrm{D}$-measurements of the droplet surface. ${ }^{24}$ These articles consistently showed that the spreading of an impacting mm-sized droplet can be described sufficiently by the Reynolds number Re $=\rho D_{0} V_{0} / \mu$ with liquid density $\rho$, dynamic viscosity $\mu$, and the Weber number We $=$ $\rho D_{0} V_{0}{ }^{2} / \sigma$, with surface tension $\sigma$. In addition, the droplet-air interaction was found to be crucial in the initial stages of droplet impact, ${ }^{17,19,32,38-41}$ as schematically shown in Fig. 1b. Prior to impact, the gap between the droplet and the surface becomes extremely slender, and the escape of the gas from this gap is strongly limited by viscous dissipation in the gas. The trapped gas forms a film between the droplet and the substrate. This film causes a "dimple" in the droplet surface due to the pressure building under the falling droplet, and prevents immediate contact between the droplet and the substrate, see Fig. 1b. Due to the dimple, the (axisymmetric) minimum of the air film thickness is found off-axis. Eventually, continued thinning of the air film results in contact between the droplet and the substrate, and a wetting front starts running inwards and outwards from the initial, ring-shape contact (indicated by small arrows). The dynamics of the entrapped air can be complex, ${ }^{\mathbf{4 2}-44}$ but usually the air entrained by the dimple forms a small bubble inside the droplet. ${ }^{\mathbf{1 4 4 4 , 4 5}}$ The dimple- and bubblesizes were quantified experimentally, numerically and theoretically, using the Reynolds-, Weber-, and Stokes-numbers as control parameters, with Stokes number St $=\rho D_{0} V_{0} /\left(2 \mu_{\mathrm{g}}\right)$, in which $\mu_{\mathrm{g}}$ is the gas viscosity. ${ }^{19}$

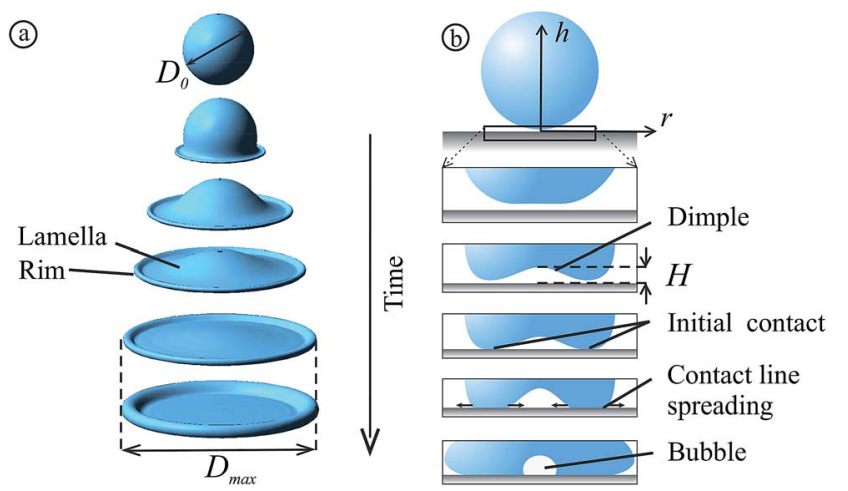

Fig. 1 Overview of the different stages of droplet impact. (a) A droplet with diameter $D_{0}$ and velocity $V_{0}$ is impacting onto a flat smooth surface (only liquid shown). During its spreading into a lamella, a rim is formed. (b) Droplet-substrate interaction in the initial stage of impact, after ref. 17. The rectangle in the top image is enlarged in the subsequent images, showing snapshots of the cross-section of the droplet. Initially the droplet flattens and forms a dimple, due to compression of the ambient gas between the droplet and the surface. After the initial contact is made, the air under the dimple is entrained and contracts into a bubble.
Can these results for mm-sized droplets provide any insight into micro-scale impacts? As discussed, the parameter space of the impact problem is huge, with the droplet diameter, velocity, viscosity, surface tension, and ambient pressure being the most relevant parameters. A cross-section of this parameter space (the diameter-velocity sub-space) is shown in Fig. 2a. Even in this limited cross-section the phenomenology of the impact problem is extremely rich. Consider for example (c) the entrainment of an air bubble by an impacting microdroplet; $^{\mathbf{1 4}}$ (d) a microdroplet during its impact on a flat surface ${ }^{31}$ (e) the development of a thick rim, which for different impact conditions can develop an instability shown in Fig. $2 f^{33,46}(\mathrm{~g})$ a droplet bouncing back from a superhydrophobic surface; $;^{21,47,48}$ and (h) splashing of an ethanol droplet on a flat surface. ${ }^{32}$ Assuming the relevance of certain forces and processes, one achieves a dimensionless representation of the parameter space as shown in Fig. $2 \mathrm{~b}$ (in terms of the Weber- and Reynolds numbers; the Stokes number is the third dimensionless parameter). This shows that the dimensionless control parameters for microdroplet impact coincide with experiments performed for mm-sized droplets (which are performed at a higher viscosity and lower velocity as compared to micrometer-sized droplets, see for example ref. 18 and 25). The impact problem is scale-invariant, which means that the mm-droplet experiments can be used to obtain accurate predictions for the micro-scale experiments. However, it is not obvious that this scale-invariance must hold. For example the wetting properties of sessile macro- and microdroplets can be rather different, ${ }^{49}$ due to different contact angles at the macro- and micro-scale. Also, the other dimensionless parameters may become relevant at small scales. Thus, it is a priori unclear whether different dimensional representations of the same point in the dimensionless subspace of the impact problem indeed show the same behavior - this depends on whether the assumptions regarding the relevant processes and forces are correct.

In this work we provide a comprehensive analysis of microdroplet impact employing novel experimental and numerical methods discussed in Section II. We visualize for the first time the impact of fast $\left(V_{0} \gg 10 \mathrm{~m} \mathrm{~s}^{-1}\right)$ microdroplets at high resolutions in Section III A, using an interferometric approach to extract the 3D-profile of the droplet surface during spreading at sub-micron resolution. In the subsequent sections we use the numerics and experiments to analyze the spreading dynamics, the air entrainment, the boundary layer flow, the development of a rim, the maximal spreading, and the splashing threshold, and compare these results to those obtained for mm-scale droplets.

\section{Droplet generation, visualization, and modeling}

\section{A. Droplet generation}

To obtain high-resolution images of fast droplet impact on a glass substrate, we create highly reproducible microdroplets (both temporally and spatially), analogous to what was done in 

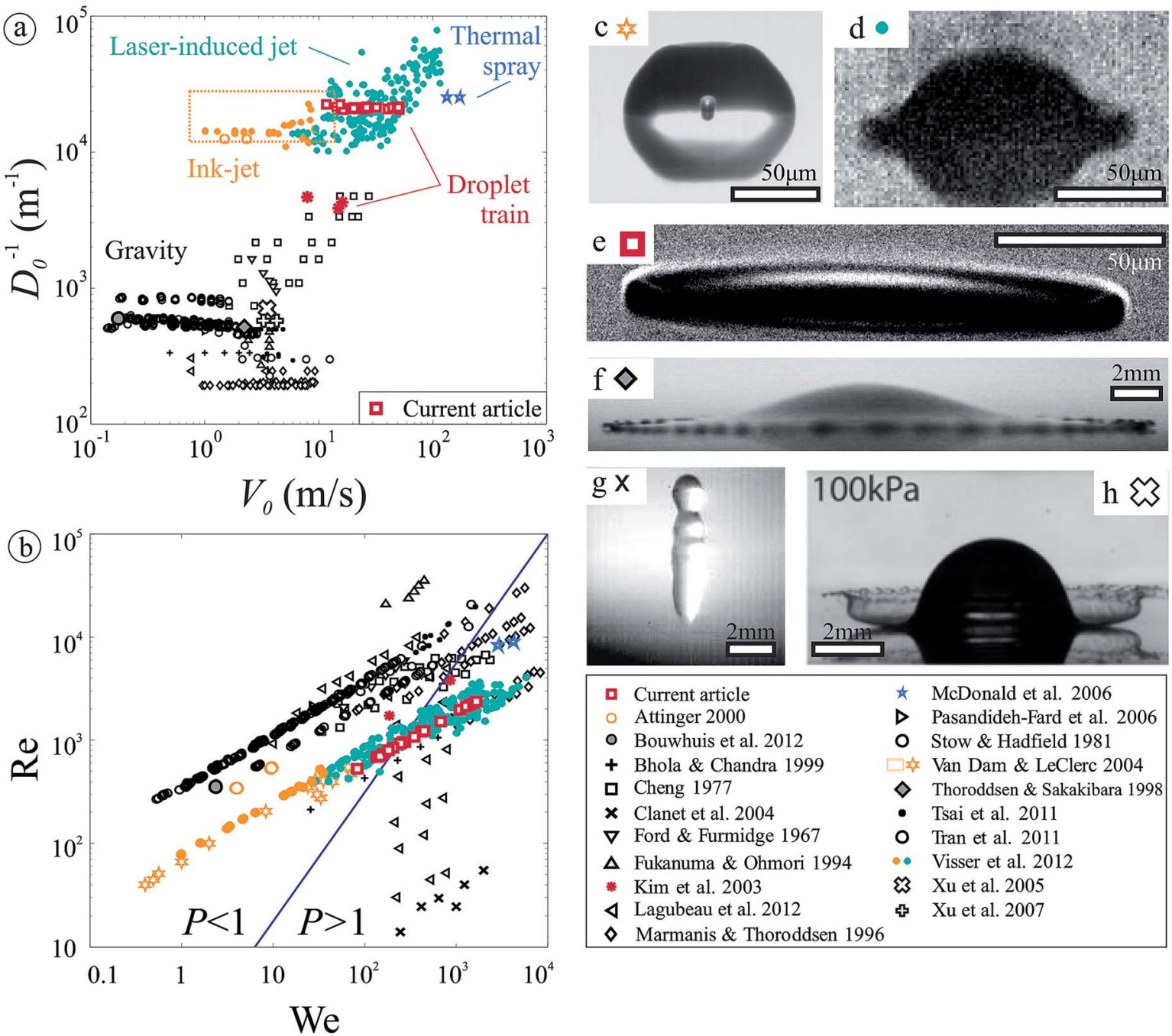

\begin{tabular}{|c|c|c|c|}
\hline व & Current article & $\star$ & McDonald et al. 2006 \\
\hline 0 & Attinger 2000 & 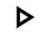 & Pasandideh-Fard et al. 2006 \\
\hline 0 & Bouwhuis et al. 2012 & 0 & Stow \& Hadfield 1981 \\
\hline+ & Bhola \& Chandra 1999 & $\square$ & Van Dam \& LeClerc 2004 \\
\hline$\square$ & Cheng 1977 & $\diamond$ & Thoroddsen \& Sakakibara 1998 \\
\hline $\mathbf{x}$ & Clanet et al. 2004 & - & Tsai et al. 2011 \\
\hline $\boldsymbol{\nabla}$ & Ford \& Furmidge 1967 & 0 & Tran et al. 2011 \\
\hline$\Delta$ & Fukanuma \& Ohmori 1994 & $\bullet$ & Visser et al. 2012 \\
\hline & Kim et al. 2003 & $\mathfrak{m}$ & $\mathrm{Xu}$ et al. 2005 \\
\hline$\triangleleft$ & Lagubeau et al. 2012 & \& & Xu et al. 2007 \\
\hline$\diamond$ & Marmanis \& Thoroddsen 1996 & & \\
\hline
\end{tabular}

$\diamond$ Marmanis \& Thoroddsen 1996

Fig. 2 Overview of experimental work on droplet impact, from ref. 3, 5, 14, 15 and 18-33, showing the phase of inverse diameter versus velocity (a) and the Reynolds number versus the Weber number (b). The line indicates the separation between viscosity-and surface tension-dominated regimes (under versus above the line, respectively) of droplet spreading as proposed in ref. 21, and discussed in Section III F. Figures (c-f) illustrate the wide range of outcomes of droplet impact. Microdroplet impact on a flat surface is shown for water at room temperature in (c-e), illustrating the entrainment of an air bubble at low-velocity impact (c), ${ }^{14}$ the limited resolution obtained by high-speed camera imaging at high-velocity impact (d), ${ }^{31}$ and the development of a rim (e) (current work). Figures ( $\mathrm{f}-\mathrm{h}$ ) are obtained for mm-sized impact, illustrating rim instabilities (f), ${ }^{28}$ bouncing (g), ${ }^{21}$ and splashing (h). ${ }^{32}$

ref. 15, 50 and 51. Fig. 3 shows an image of our setup. We first produce a liquid jet by pumping distilled water through a $\sim 20$ $\mu \mathrm{m}$ orifice. Using an acoustic element the jet is vibrated at a controlled frequency, forcing Rayleigh break-up of the jet into a train of monodisperse droplets. By applying a positive electrical pulse of duration $\sim \tau$ to the charging electrode, the tip of the jet is temporarily charged negatively at the moment of break-up, and a charged droplet is created. When traveling in the electric field between the deflection plates, this droplet is slightly deflected $\left(\theta<1^{\circ}\right)$. Catching away the droplet train then results in impact of a single droplet on the impact plate, at a velocity controlled by the jet velocity. Three surfaces were used to assess the influence of the macroscopic advancing contact angle $\theta$ for droplet spreading: clean glass, with $\theta=23^{\circ}$; RainX, a windshield-repellency coated glass surface (brand: RainX) with $\theta=90^{\circ}$, and a hydrophobized aluminium oxide surface ${ }^{52}$ with $\theta=135^{\circ}$. To prevent charge build-up, the capillary tube, the impact plate, and the reservoir were grounded. The parameter space that was scanned with the current setup is shown in Fig. 2a (all experiments were performed in air under standard conditions).

\section{B. Droplet visualization}

To visualize the droplet from the side, a double-shutter camera (Sensicam QE, PCO AG, Germany) was used. A 6 ns pulse from a dual-flash ND:YAG laser (Quantel, France) provided bright-field illumination. To prevent fringes, coherence was removed by inserting a fluorescent plate in front of the laser. A bottom view was realized similar to the 


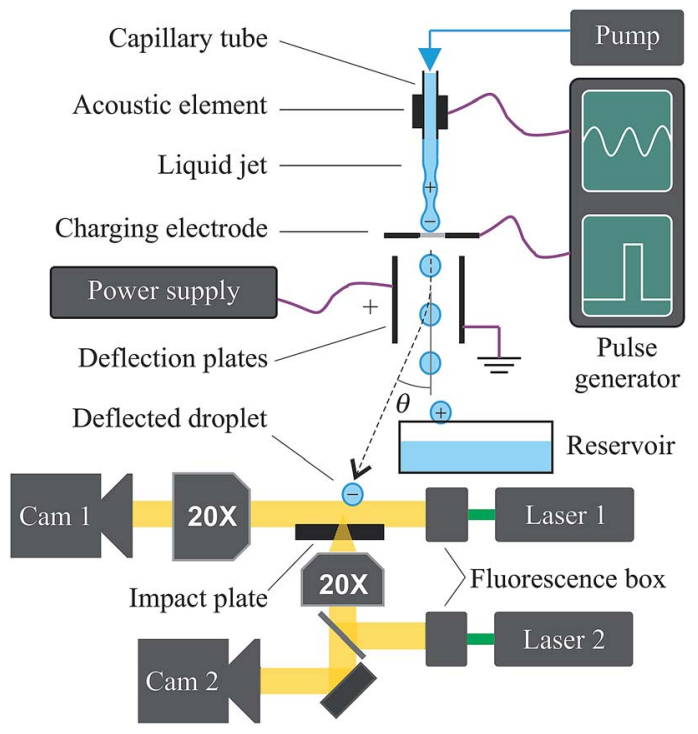

Fig. 3 Setup. A liquid jet is created by pumping the liquid through a 20 $\mu \mathrm{m}$-sized orifice. The jet is acoustically oscillated, to force its break-up into a train of monodisperse droplets. A high-voltage pulse is applied to a charging electrode, just before break-up into a droplet. This charges the jet tip, resulting in a single, charged droplet. This droplet is deflected from the non-charged droplet train by an electrical field. Catching away the droplet train then results in a single, highly reproducible impact on the impact plate. Synchronized side- and bottom views are used for visualization. side view. A half-mirror was added to achieve co-axial, darkfield illumination. The camera- and laser timings were synchronized for the side- and bottom-views. All temporal delays were controlled using pulse generators (Berkeley Nucleonics Corporation, model 575).

\section{Determination of droplet size, velocity, and moment of impact}

For each experimental setting, the droplet size and velocity were determined as displayed in Fig. 4. From two side-view images of the in-flight droplet, indicated as $1 \mathrm{~A}$ and $1 \mathrm{~B}$ in Fig. 4a, the droplet position was obtained by automated image analysis in MATLAB (MathWorks Inc., Natick, MA, 2013). As the delay between the frames $\Delta t$ is known, the spatial difference $\Delta y_{1}$ readily provides the droplet velocity $V=\Delta y_{1} / \Delta t$. For each experimental setting, at least 10 image pairs were processed to obtain a velocity histogram as plotted in Fig. 4b. From this histogram, the median velocity provides the per-experiment velocity $V_{0}$. In all cases, the velocity has a typical standard deviation of $0.01 V_{0}$. The droplet diameter was obtained from frames $1 \mathrm{~A}$ and $1 \mathrm{~B}$ of the velocity calibration measurements and each first frame of the impact experiments (frame $2 \mathrm{~A}$ ), resulting in $\sim 150$ diameter measurements for each experimental setting. A histogram of the droplet diameter is plotted in Fig. 4d. Again the median provides $D_{0}$ with a typical standard deviation of $0.01 D_{0}$.
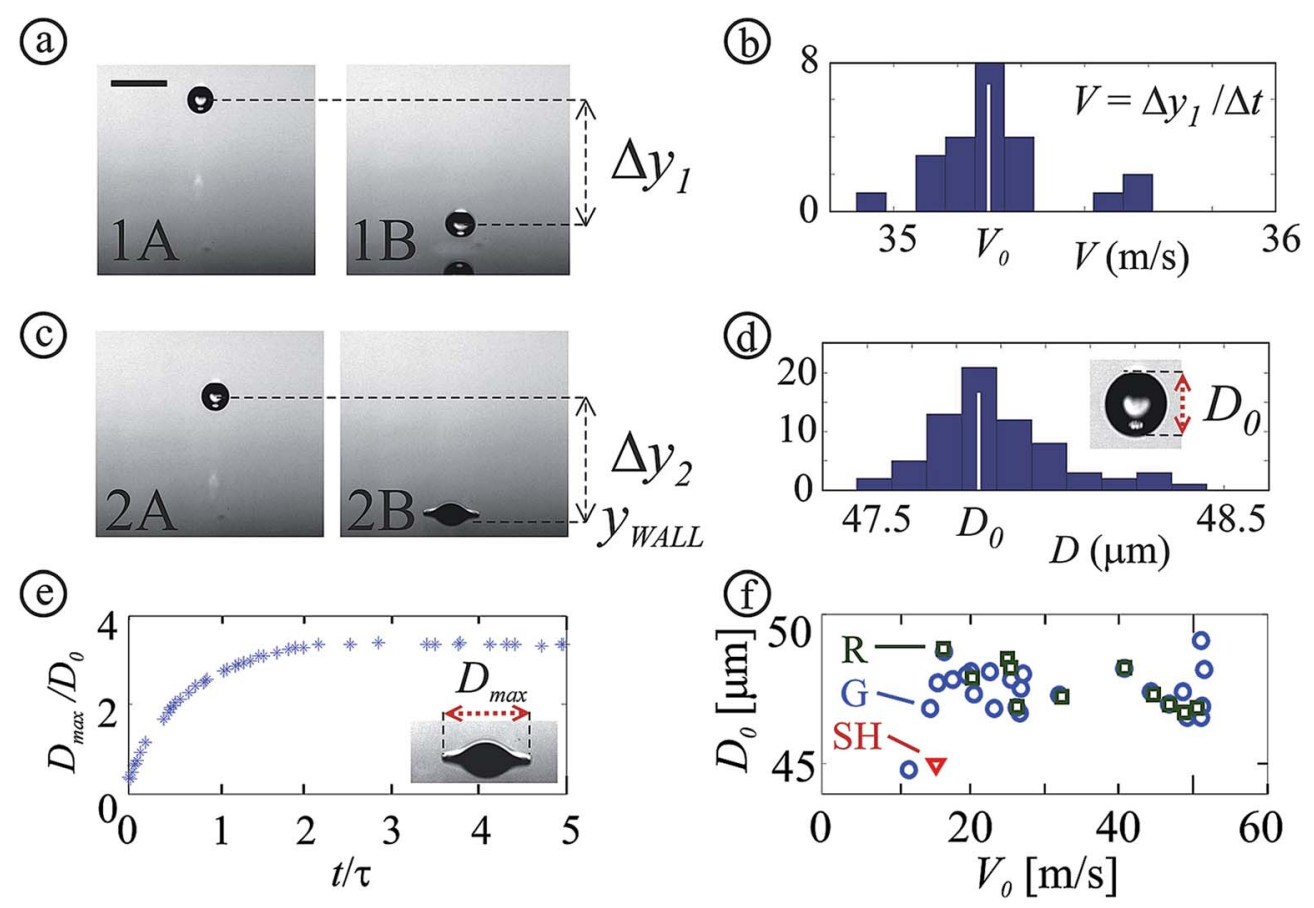

Fig. 4 Determination of droplet size and velocity. All images shown are for equal droplet generation settings. (a) Two in-flight frames are used to determine the droplet translation $\Delta y_{1}$. The translation is used to obtain the droplet velocity. The scale bar indicates $100 \mu m$. (b) Velocity histogram. The median provides the impact velocity $V_{0}$. Here and in (d), note the scale of the velocity axis: the distribution is very narrow. (c) Droplet captured in-flight (frame 2A) and during spreading (frame 2B). Frame A is used to collect the diameter $D$ for each experiment. (d) Diameter histogram. The median provides the droplet diameter for each experimental setting, $D_{0}$. (e) Example curve of $D_{\max } / D_{0}$ is plotted versus time. The maximum spreading diameter $D_{\max }$ is determined from frame 2B. (f) Parameter space of $D_{0}$ and $V_{0}$. The markers indicate the surface type (G: glass $\left(\theta=23^{\circ}\right)$, R: RainX $\left(\theta=90^{\circ}\right)$, SH: superhydrophobic $\left.\left(\theta=135^{\circ}\right)\right)$. 


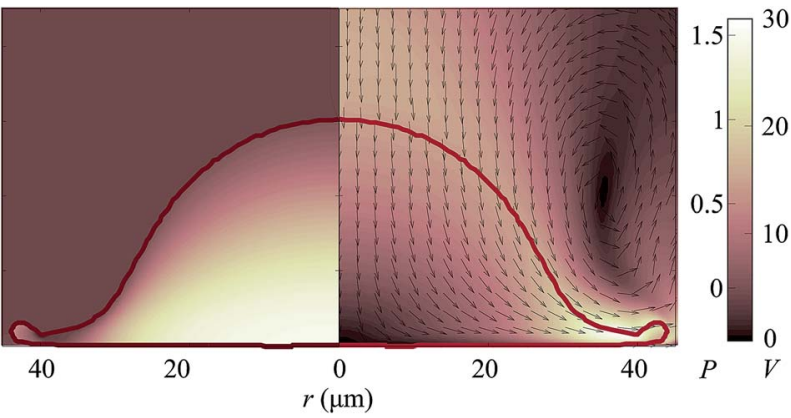

Fig. 5 Simulated droplet impact. Left half: pressure field; the color map indicates the pressure $P$ in $10^{5} \mathrm{~Pa}$. Right half: velocity field, in which the arrows and the colors respectively indicate the flow direction and flow velocity (in $\mathrm{m} \mathrm{s}^{-1}$ ). Simulation parameters: $D_{0}=50 \mu \mathrm{m}$ and $V_{0}=17 \mathrm{~m} \mathrm{~s}^{-1}$, corresponding to $\mathrm{We}=195$ and $\mathrm{Re}=836$. The snapshot is taken at $t / \tau=0.42$.

An example impact experiment is shown in Fig. 4c, where frame B shows a clear image of the impacting droplet. However, the arrival time of the droplet has an uncertainty of $\sim 1 \mu$ s due to the $1 \%$ uncertainty in the droplet velocity. This is similar to the typical impact time $\tau$, thus a more accurate time determination is required to resolve the impact dynamics. Therefore, for each image pair, we first calculate the time difference between the first droplet-substrate contact and frame A as $t_{\mathrm{A}}=-\tau / 2-\Delta y / V_{0}$, with $\Delta y$ the distance between the droplet and the substrate. This yields a negative value, as time $t=0 \mathrm{~s}$ is defined as the moment of impact. The time of frame $\mathrm{B}$ is $t_{\mathrm{B}}=t_{\mathrm{A}}+\Delta t$, with $\Delta t$ the frameto-frame delay as set with the pulse generator. The per-frame time uncertainty using this method is $100 \mathrm{~ns} \ll \tau$, i.e. sufficient for our measurements. To scan the temporal axis of the full impact, this procedure is repeated $\sim 150$ times for each experimental setting, with different delays. High-speed movies of the side- and bottom view are constructed for each experiment

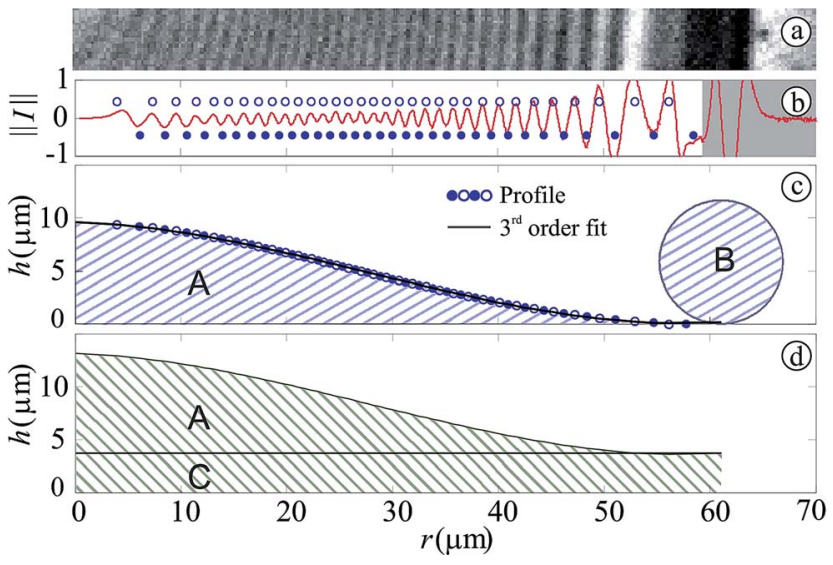

Fig. 7 Bottom view analysis. (a) Details of Fig. 6d, clearly showing fringes. (b) Radially-averaged intensity map of the bottom view image. The open and closed markers show the radial positions where respective bright and dark fringes were detected. The shaded area is not used for analysis: (c) the rim profile determined from the fringes is shown by the open and closed markers, corresponding to figure (b). The black line indicates a third-order fit. Integration of area $A$ provides the volume contained under the profile, which is less than the original drop volume. Area $B$ corresponds to the rim volume if the rim would contain all excess volume of the droplet. (d) Area $C$ corresponds to the excess liquid if it would be fully contained under the droplet, resulting in an offset of the droplet profile equal to the height of area $C$.

(example movies are provided for control parameters listed in Table $\mathrm{S} 1$ and Fig. S1†).

\section{Numerical methods}

Simulations were done using the open-source flow solver Gerris, ${ }^{53}$ which numerically solves the incompressible NavierStokes equation on a non-uniform flow-adaptive grid. The interface-tracking volume of fluid (VOF) implementation in Gerris makes it well-suited for problems involving two phases
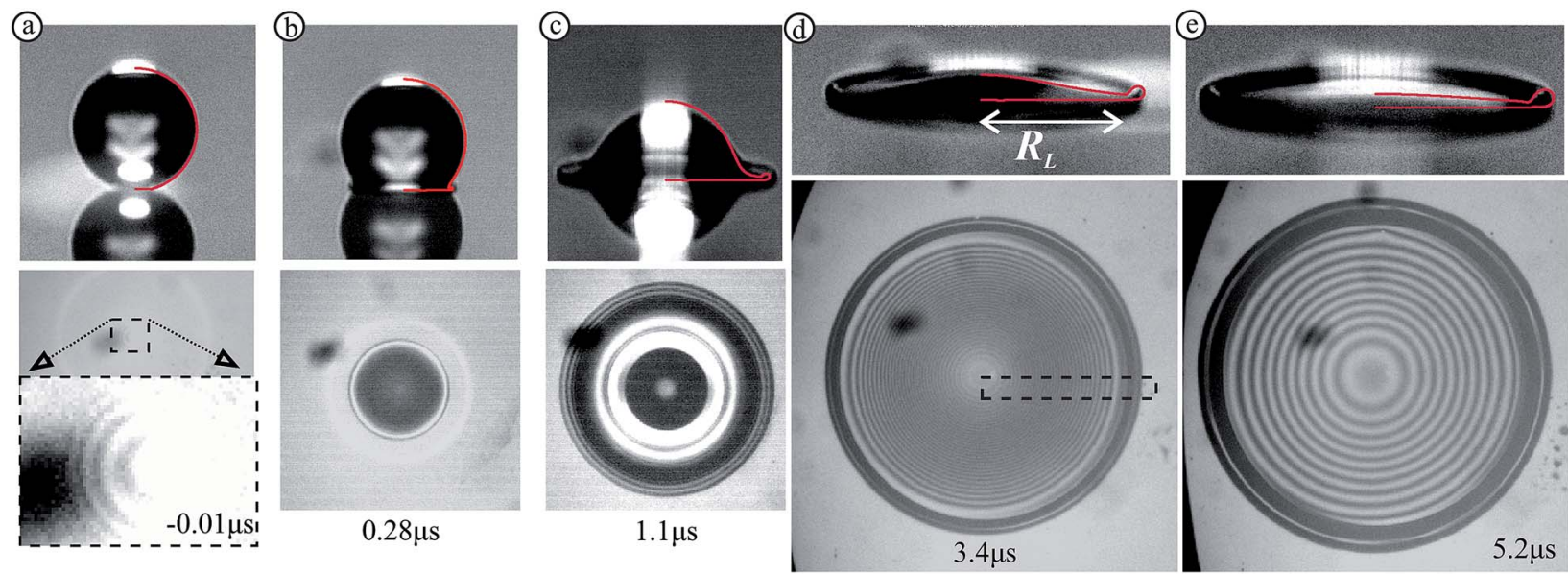

Fig. 6 Side- and bottom-view snapshots of a water droplet impacting onto dry glass for five different times (displayed in the bottom-view images). The side views include the numerically obtained droplet contour (red line, plotted in the right half of the image, see main text). The dark, blurred spots in the bottom view are caused by dirt on the bottom side of the glass. The inset in figure (a) shows air-fringes (explained in main text). The dashed rectangle in figure (d) is magnified in Fig. 7a. Impact parameters: $D=49.5 \mu \mathrm{m}$ and $V=16.9 \mathrm{~m} \mathrm{~s}^{-1}$, corresponding to Re $=836$, $\mathrm{We}=196$, and $\mathrm{St}=2.2 \times 10^{4}$. 
with a high density ratio and interfacial tension (water and air). In our axisymmetric simulations we take a uniform grid inside the droplet with typically 340 points across the diameter (i.e. $0.15 \mu \mathrm{m}$ grid cell size for a $D_{0}=50 \mu \mathrm{m}$ droplet). The interface of the drop is adaptively discretized, ensuring that the curvature per cell does not exceed $5 \%$ of the droplet radius. The minimum resolution is limited to the resolution inside the droplet. Outside the droplet the grid is adapted according to the norm of the vorticity, with a maximum grid size equal to that of the droplet. The size of the (squared) simulation domain is 3 droplet diameters, with outflow boundary conditions on the top and left edge, no-flow conditions on the bottom edge and symmetry conditions on the right edge (which represent the $z$ axis along which the droplet approaches the surface). During the downward impact, the air-layer between the droplet and the bottom surface is given extra resolution to prevent the VOFinterface from coalescing with the wall (which also allows for bigger time steps). In most simulations there remains a minimum gap of about $20 \%$ of a droplet grid cell (i.e. $30 \mathrm{~nm}$ ) between the bottom of the domain and the droplet interface. The viscosity and density do not sharply change over the interface, which means that the droplet is effectively in contact with the no-slip bottom edge at these points (with a $180^{\circ}$ contact angle at the contact line).

Example results of the model are shown in Fig. 1a and 5. The model is validated versus our measurements in Fig. 6, 8, 9, and 14 , as will be discussed in the corresponding subsections of this article.

\section{Results and discussion}

\section{A. Droplet profile}

From the side- and bottom view snapshots shown in Fig. 6, we can identify different stages of the droplet impact. Image $6 \mathrm{a}$ shows the droplet just before impact. Subsequently, the droplet spreads over the surface into a lamella. As is visible in images $6 \mathrm{c}$ to $6 \mathrm{e}$, a rim is formed at the edge of the sheet. After reaching its maximal diameter, the droplet retracts (for hydrophobic surfaces) or the contact line pins and the droplet comes to rest after some capillary oscillations. As we focus on the impact dynamics, these stages are not shown here.

Prior to impact, the bottom view reveals faint fringes (Fig. 6a). These fringes are due to the air film between the droplet and the substrate, and will be discussed in subsection III C. In the later stages of droplet spreading, the bottom view again reveals clear fringes (Fig. $6 \mathrm{~d}$ and e). These fringes are due to interference between the top droplet-air interface and the glass or droplet bottom.

We now analyze the droplet surface profile from the fringes in Fig. 6d, which have been magnified in Fig. 7a. After radial averaging, the black and white fringes result in the intensity profile shown in Fig. $7 \mathrm{~b}$. The local minima and maxima of this curve were automatically detected using MATLAB and are plotted as open and closed circles, respectively. As the droplet thickness is monotonically decreasing in height within the lamella radius (indicated as $R_{\mathrm{L}}$ in Fig. 6d), each black-to-white fringe transition is associated with a quarter-wavelength step down in height. The resulting profile is indicated by the dotted curve in Fig. 7c, where the minimum is set to zero as an initial value. However, the fringes only provide the slope of the droplet surface, thus the actual lamella thickness remains to be determined.

To find the actual lamella thickness we calculate the volume contained within the profile plotted in Fig. 7c, by fitting a thirdorder polynomial to it (black line) and integrating the resulting curve assuming axisymmetry. This volume, corresponding to area " $A$ " in Fig. 7c, is much smaller than the actual droplet volume. This is not surprising, as a part of the droplet volume is contained in the rim, and the lamella must have a non-vanishing thickness everywhere. If we assume that the rim contains all excess volume, the profile drawn in Fig. 7c is obtained, with a large rim and a vanishing lamella thickness just inside the rim. Alternatively, assuming that all excess volume is present below the profile, we obtain the profile without a rim as plotted in Fig. 7d. The actual profile, which lies in between these two limiting cases, can be obtained with higher precision from the side view. Using the rim size given by the circle in Fig. 8a and integrating this to obtain the rim volume, both the rim radius and the film thickness are fixed. In Fig. 8b, the result is plotted using MATLAB (the lighting was set to make the images (a) and (b) correspond, and a mirror image of the droplet is plotted (in black) below the droplet). Finally, in Fig. 8c, the derived profile is shown to be in good agreement with the numerical result. Theoretical profiles were derived for inviscid impact. ${ }^{36,54}$ However, since viscosity results in the formation of a boundary layer which affects the film thickness for impact on solid surfaces, ${ }^{54}$ these predictions deviate from our results (in particular, a much thinner lamella with a far larger radial extension is obtained from theory) and are therefore not included. As the numerical results are based on the Weber, Reynolds, and Stokes-numbers alone, the good overall agreement with the experimental droplet profiles provides first evidence for the scale-invariance of the impact events studied

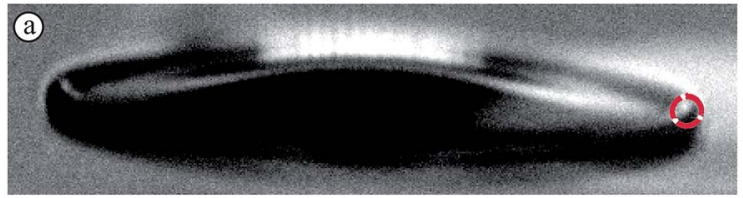

(b)
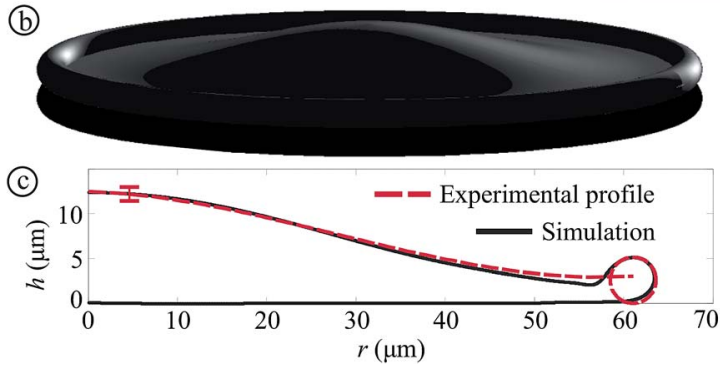

Fig. 8 Bottom view analysis combined with side view and simulation, for the droplet analyzed in Fig. 7. (a) Side view image. In red the rim size is shown, which was used for the analysis of the bottom view images as explained in the main text. (b) Side-view image reconstructed from the fringes in the bottom-view. (c) Rim profiles determined from experiment and simulation. The red vertical line indicates the experimental error. 
(a)

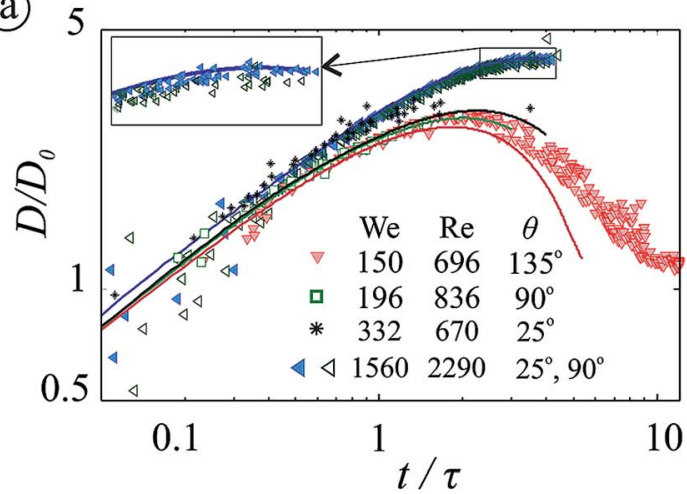

(c)

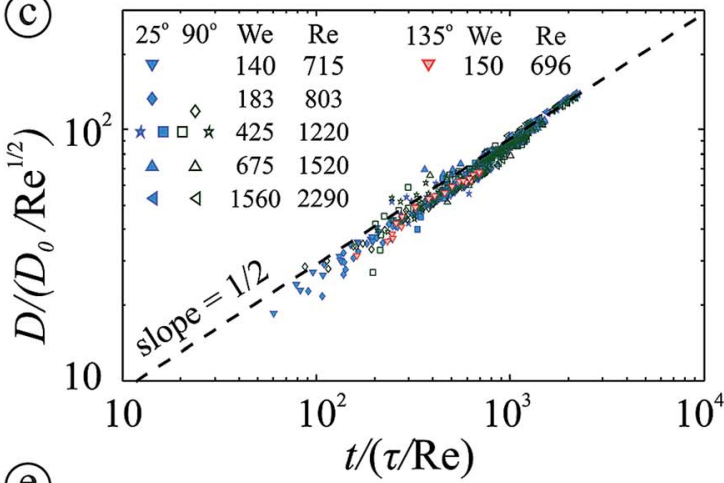

(e)

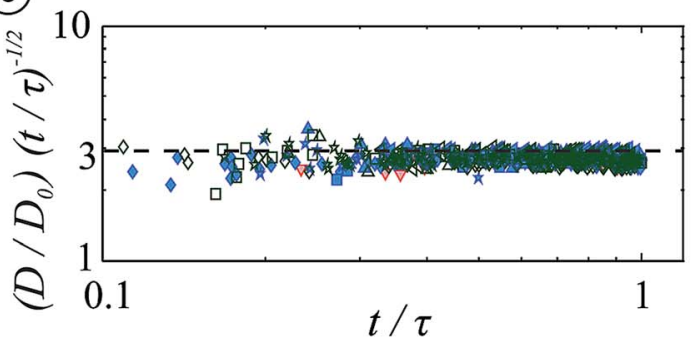

(b)
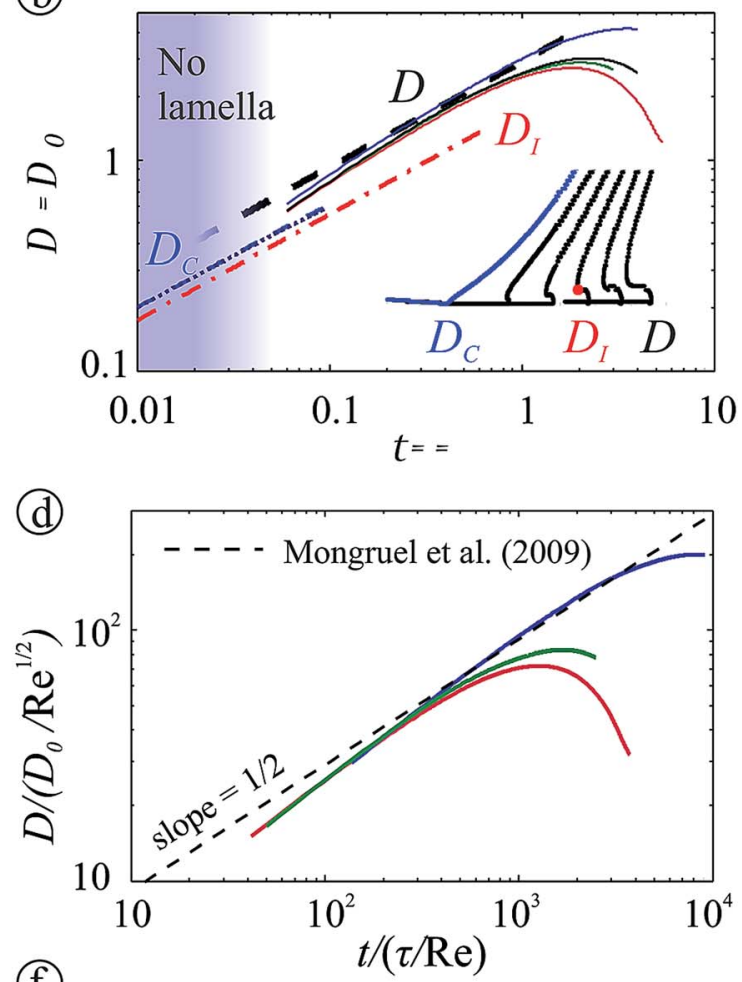

(f)

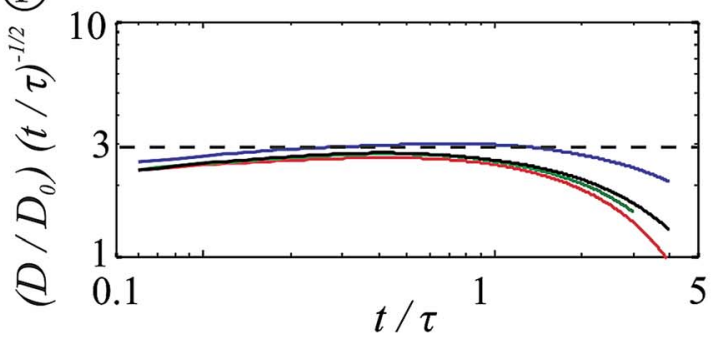

Fig. 9 Normalized spreading diameter versus time. Left: (mainly) experiments. Right: numerical results. (a) Comparison between measurements (markers) and simulations (lines) for three different contact angles. The black stars represent $\mathrm{mm}$-sized droplet data from ref. 55 ( $D_{0}=2.7 \mathrm{~mm}$ and $V_{0}$ $=2.6 \mathrm{~m} \mathrm{~s}^{-1}$, We and Re in the legend). The spreading is contact-angle independent and good agreement with the numerical results is obtained, as illustrated by the collapsing curves in the inset. Final retraction of the droplet is observed only for the strongly hydrophobic surface $\left(\theta=135^{\circ}\right)$ and in the simulations $\left(\theta=180^{\circ}\right.$ ). (b) Development of the contact diameter before lamella ejection $D_{\mathrm{C}}$ (blue dash-dotted line), the inflection diameter $D_{1}$ (red dashed line), and the maximal lamella diameter $D$ (black dashed line); solid lines show the numerical results corresponding to figure (a). The colorgradient indicates the predicted transition towards the lamella regime, ${ }^{56}$ as discussed in the main text. A time series of the droplet shape is shown in the right-bottom corner of the figure, illustrating the contact diameter $D_{C}$, the inflection diameter $D_{1}$, and the maximal lamella diameter $D$. (c) Normalized lamella spreading versus normalized time. The experimental data (shown for $t / \tau<1$ ) collapse to the model proposed by Mongruel et al. ${ }^{57}$ (eqn (2), shown as a dashed line in figures (c to f)) within the experimental error. (d) The numerical results (solid lines) collapse to theory ${ }^{57}$ at intermediate times. For small times the lamella is not fully developed; in the late regime surface tension and viscosity limit the spreading. (e $\& \mathrm{f}$ ) The corresponding compensated plots $\left(D / D_{0}\right)(t / \tau)^{-1 / 2}$ versus $t / \tau$ (out of which Re cancels) for figures (c $\& d$ ).

here. In the following sections we will discuss the different aspects of the impact in detail.

\section{B. Spreading dynamics}

In Fig. 9a, the spreading dimensionless diameter $D / D_{0}$ is plotted as a function of dimensionless time $t / \tau$, for different Weber- and Reynolds numbers (indicated by symbol shape) and for impact on surfaces with different wettabilities (indicated by marker color). For $t / \tau \leqslant 3$, we found a negligible influence of the contact angle on the spreading radius, as was also observed for mm-sized droplets. ${ }^{55}$ Good agreement between our measurements and our numerical model (solid lines) is reached. In fact, even better agreement with the numerical results is obtained than with mm-sized droplets, as in measurements with mm-sized droplets (ref. 55, plotted as *) slightly stronger spreading than in our numerical simulations is seen, for unknown reasons.

To compare our results with the literature, we require a more detailed picture of the spreading dynamics of the droplet. The impact can be divided into three different phases, of which the first two are depicted by the inset in Fig. 9b: 


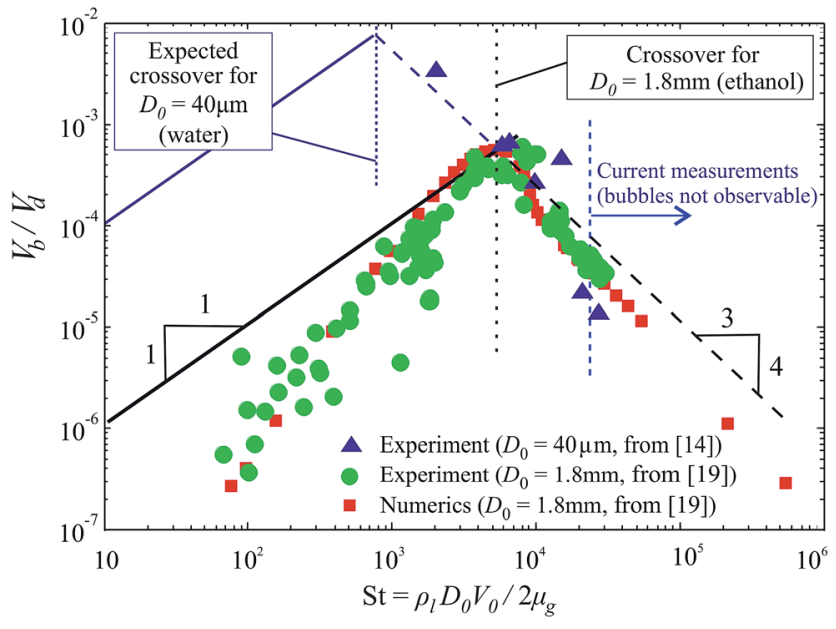

Fig. 10 Dimensionless volume of the bubble entrained during droplet impact versus the Stokes number (St). For microdroplets, the bubble volume was measured by van Dam and Le Clerc (2004) ${ }^{14}$ and re-cast here (blue markers), as for the current experiments the bubble size is smaller than the optical resolution of the setup. For comparison to air entrainment by $\mathrm{mm}$-sized droplets, the experimental and numerical results from Bouwhuis et al. (2012) ${ }^{19}$ are included (green and red markers). The lines show scaling laws proposed for the capillary air entrainment regime (low-St, solid lines, from ref. 19) and the inertial air entrainment regime (high-St, dashed lines, from ref. 19 and 60). The vertical blue and black dashed lines indicate the crossover from the capillary to the inertial regime, for micro- and $\mathrm{mm}$-sized droplet impact, respectively.

- The initial phase, in which the bulk of the droplet maintains its initial velocity and no lamella is ejected (blue line in the inset of Fig. 9b). The droplet-substrate contact diameter $D_{\mathrm{C}}$ was initially described assuming compression of the liquid in the droplet (i.e. lack of lamella ejection), resulting in $D_{\mathrm{C}} / D_{0}=2 \sqrt{t / \tau-(t / \tau)^{2}}$ from geometry arguments. ${ }^{55} \mathrm{~A}$ modification was made by assuming incompressible flow from the droplet into a disk-shaped lamella, ${ }^{\mathbf{1 4 5 8}}$ but for $t / \tau \ll 1$ this result collapses to the aforementioned result from ref. 55. Recently, the radial position of the inflection point $D_{\mathrm{I}}$ was calculated using Wagner's theory, ${ }^{59}$ resulting in $D_{\mathrm{I}}=\sqrt{3 t / \tau}$, and proposed as the initial wetting velocity. ${ }^{56}$ As the transition to the next regime (where the lamella is ejected) occurs on a time scale of order $\tau / 100$, we could not yet study this regime. To conclusively measure the dynamics in this regime (also for mm-sized droplets), high-resolution measurements would be required, in particular in view of the small differences between the various predictions.

- The lamella phase. A lamella is ejected when its velocity exceeds the wetting velocity observed in the initial phase. ${ }^{56,57}$ This occurs at the lamella ejection time $t_{\mathrm{e}}$, which was recently modeled $^{56}$ by the solution of the implicit equation

$$
\sqrt{3} / 2 \mathrm{Re}^{-1}\left(t_{\mathrm{e}} / \tau\right)^{-1 / 2}+\mathrm{We}^{-1}=C\left(t_{\mathrm{e}} / \tau\right)^{3 / 2},
$$

with prefactor $C=1.2$. For our control parameters this yields a value of $0.02<t_{\mathrm{e}} / \tau<0.04$, illustrated by the transition from the shaded (blue) region to the white region in Fig. 9b. The lamella spreading velocity and radial extension were obtained by equating the inflow from the drop and the outflow into the lamella (modeled as a circular disc with an empirically obtained thickness): ${ }^{57}$

$$
\frac{D_{\mathrm{L}}(t)}{D_{0}}=2.9 \sqrt{\frac{t}{\tau}} .
$$

A comparison with our experimental results in this regime is provided in Fig. 9c, where the axes were chosen to allow direct comparison to ref. 57 . The experimental results are limited to $4 t_{\mathrm{e}}<$ $t<\tau$, ensuring that only the lamella regime is captured. The model and experiments agree quantitatively within the experimental error, again evidencing scale-invariance of the drop impact dynamics. Our numerical results (Fig. 9d) show reasonable agreement for intermediate times, but deviate for small times (where the transition from the slower initial regime results in a lower value), and for large times (due to slow-down, next item).

- The slow-down phase $(t / \tau>1)$, in which surface tension and viscosity significantly slow down the spreading lamella. This eventually stops the spreading, defining the maximum spreading diameter as discussed in subsection III F.

\section{Droplet-air interaction}

As discussed in the Introduction and shown in Fig. 1b, the gas in the gap between the droplet and the surface forms a dimple
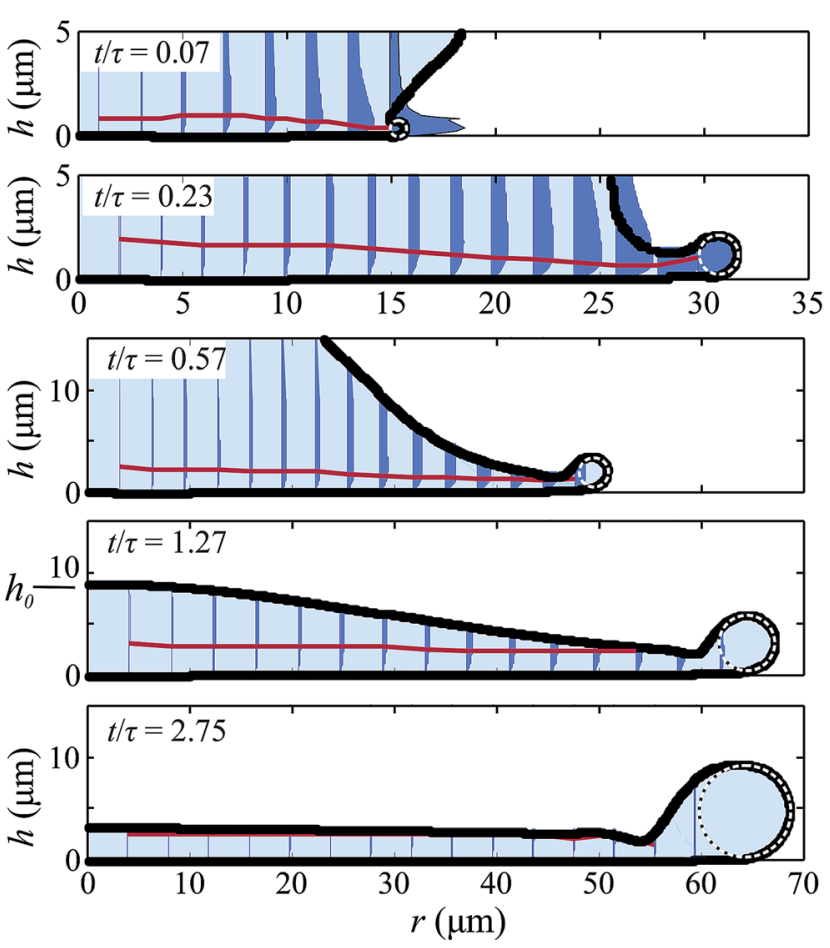

Fig. 11 Snapshots of the simulated cross-section of the droplet, for $\mathrm{We}=196$ and $\operatorname{Re}=836$. The black line indicates the droplet contour, the blue shading indicates the liquid, and the radial velocity components are plotted as filled areas (blue). The red line indicates the boundary layer $\delta(r, t)$. The white dotted lines show the fitted rim profile. The parameter $h_{0}$ indicates the center height of the droplet, as shown in the left side of the fourth image. Note the different scaling of the upper two images, allowing for a more detailed view. 

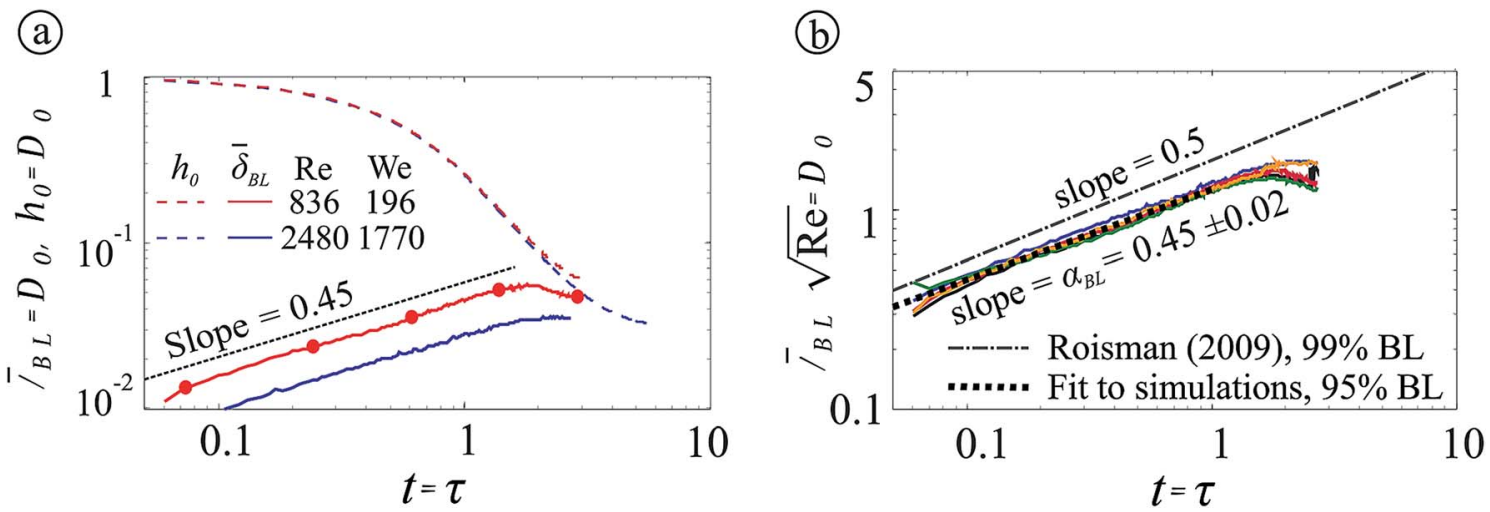

Fig. 12 (a) Simulated space-averaged boundary layer thickness versus time for two (Re, We) pairs (in red and blue, as shown in the legend), normalized by the droplet diameter. The dashed lines indicate the center height of the droplet $h_{0}(t)$. The red markers correspond to the droplet profiles as plotted in Fig. 11. Figure (b) shows the boundary layer thicknesses normalized by $\operatorname{Re}^{-1 / 2}$ (line colors indicate Reynolds and Weber numbers as in Fig. 13(b)), displaying a good collapse in this normalization.

in the droplet and "cushions" the impact. The initial contact between the droplet and the substrate therefore occurs off-axis, and air is entrained by the dimple in the droplet. As this air cannot escape, a small bubble inside the droplet is observed.

Initially we aimed to assess the air film behavior using the fringes using bottom-view interferometry, a method commonly applied to determine the air profile separating the droplet from the surface. ${ }^{17,39,61}$ However, processing the fringes shown in Fig. 6a reveals a spherical droplet profile with a curvature corresponding to the droplet size, i.e. the expected dimple was not observed. A prediction of the dimple height was provided in ref. 19 , yielding $H \sim 50 \mathrm{~nm}$ for our control parameters. As the resolution of the interference method is limited to $\lambda / 4=145 \mathrm{~nm}$ (with $\lambda=577 \mathrm{~nm}$, the illumination wavelength) the dimple profile cannot be resolved by bottom-view interferometry in the visible spectrum. Therefore, to assess the scale-invariance of the droplet-air interaction, we now discuss the bubble size.

The size of the bubble entrained by impacting droplets was determined theoretically, experimentally, and numerically for millimeter-sized droplets, ${ }^{19}$ as shown in Fig. 10. At very low Stokes numbers (i.e. slow droplets), the droplet approaches the surface so slowly that the gas can escape, and no bubble is entrained. ${ }^{62}$ For increasing (but still low) Stokes numbers, an increasingly large bubble is observed, indicated by the solid black line in Fig. 10. However, in the high-Stokes number limit, the gas is squeezed out so strongly that again very small bubbles are observed as indicated by the black dashed line ${ }^{60}$ The maximum bubble size is observed at an intermediate "crossover" Stokes number, which is plotted as a vertical dotted line in Fig. 10.

Since compressibility is likely to become important for fast micrometer-sized droplet impact, ${ }^{60,63}$ the question is whether equal dimensionless droplet sizes are observed. The bubbles entrained by microdroplet impact at lower velocities than in our experiments $\$$ were measured by van Dam and LeClerc ${ }^{14}$ and are re-cast in Fig. 10. To compare these data to the model discussed, the crossover Stokes number (which depends on the Weber- and

\$ Our current experiments (at high Stokes numbers, see Fig. 10) resulted in a bubble size below the resolution of our setup.
Reynolds numbers and will thus change for smaller droplets) was re-calculated for $D_{0}=40 \mu \mathrm{m}$ and is plotted as a blue dotted line in Fig. 10. The data from ref. 14 collapse to the prediction, and completely lie in the high-Stokes regime. Although the scale-invariance of the low-St regime remains to be addressed, this provides strong evidence for scale-invariant entrainment of air by droplet impact in the high-St regime, i.e. that no additional dimensionless numbers (apart from Re, We, and St) are required to describe the air entrainment by impacting droplets.

\section{Boundary layer development}

During impact, the radial flow over the surface results in the development of a boundary layer from the surface. Here the local boundary layer thickness $\delta_{\mathrm{BL}}(r, t)$ is defined as the height at which $95 \%$ of the maximal horizontal velocity is reached. The red lines in Fig. 11 show the boundary layer thickness derived from the simulations, which is generally well-defined (values close to the origin $(r<5 \mu \mathrm{m})$ and early times $(t / \tau<0.06)$ are inconsistent and therefore excluded). To obtain a single boundary layer thickness value for each instant in time, $\delta(r, t)$ is spatially averaged as:

$$
\bar{\delta}_{\mathrm{BL}}(t)=\frac{4 \int_{0}^{2 \pi} \int_{0}^{D(t) / 2} \delta_{\mathrm{BL}}(r, t) r \mathrm{~d} r \mathrm{~d} \theta}{\pi D(t)^{2}},
$$

with $\theta$ the angular coordinate. This spatially averaged boundary layer thickness is plotted as a function of time in Fig. 12a. The markers on the red line correspond to the snapshots in Fig. 11. A maximum boundary layer thickness is observed at $t / \tau \approx 2.5$ when the (increasing) boundary layer thickness approaches the (decreasing) droplet thickness $h_{0}(t)$ (indicated by the dashed line in Fig. 12a). This instant is important in the determination of the maximum spreading diameter, as will be discussed in detail in Section III F.

The temporal development of the boundary layer was theoretically addressed in the context of droplet impact. ${ }^{37}$ There, an analytical flow field solution for inertia-dominated inviscid flow onto a wall (long after initiation) was extended to include 
(a)

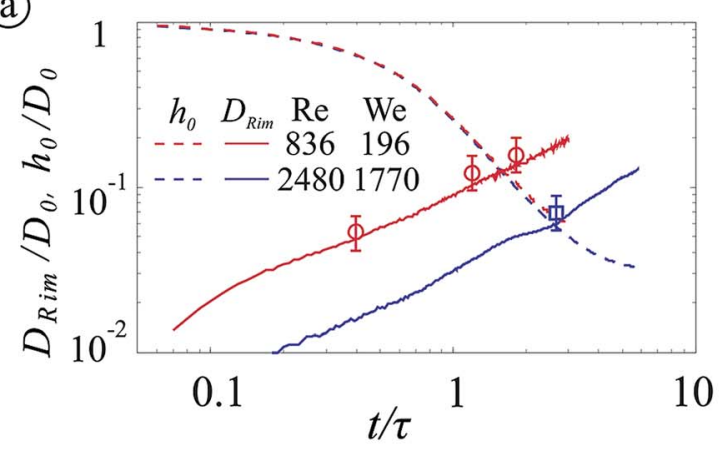

(b)

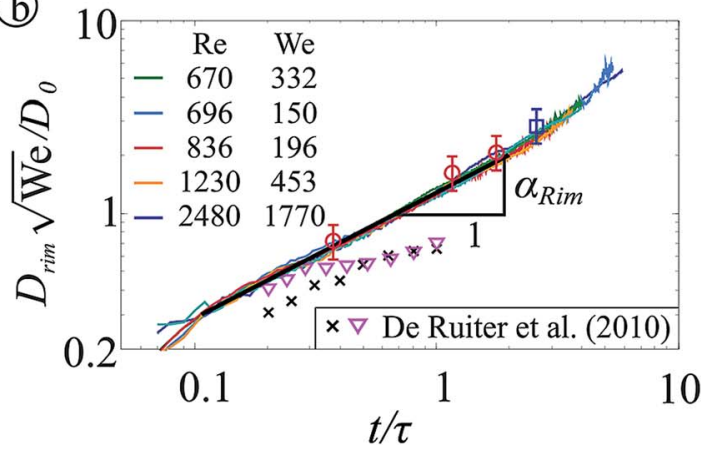

Fig. 13 Simulated rim diameter versus time, for various ( $\mathrm{Re}, \mathrm{We}$ ) pairs as shown in the legend. (a) Rim diameter normalized by the droplet diameter as a function of time normalized by the inertial time scale $\tau$. The markers correspond to measured rim diameters. The dashed lines indicate the center height of the droplet $h_{0}(t)$. Figure (b) shows the rim diameter normalized by $D_{0} \mathrm{We}^{-1 / 2}$. Fitting the rim evolution in time provides the exponent $\alpha_{\text {Rim }}=0.68 \pm 0.04$, which is indicated as a black line. Rim height measurements for mm-sized droplets are added in figure (b), from ref. 64, for $W e=59$ and $\operatorname{Re}=1340$ (black crosses, $x$ ), and $W e=185$ and $\operatorname{Re}=2360$ (magenta triangles $\nabla$ ).

viscosity and a no-slip condition on the wall. This results in the development of a boundary layer with a homogeneous thickness over the full surface area (which is surprisingly consistent with Fig. 11, showing a roughly spatially homogeneous boundary layer thickness for various instants). Based on this model, a $99 \%$ boundary layer thickness as a function of time is proposed as:

$$
\delta_{99} \approx 1.88 D_{0} \sqrt{\frac{t}{\tau \mathrm{Re}}} .
$$

which is indicated by the dash-dotted line in Fig. 12b.

The non-dimensional boundary layer thickness obtained from our simulation is plotted in Fig. 12b. For a range of Reynolds- and Weber numbers (indicated in the caption), the results collapse to a single curve which confirms the robustness of the boundary layer scaling with respect to the Reynolds number. The curve follows $\bar{\delta}_{\mathrm{BL}}(\mathrm{t}) \sim D_{0} \mathrm{Re}^{-1 / 2}(t / \tau)^{\alpha_{\mathrm{BL}}}$ with exponent $\alpha_{\mathrm{BL}}=0.45 \pm 0.02$, and lies below the prediction of ref. 37 . This latter difference is due to the different definitions of the boundary layers: we assume a $95 \%$ boundary layer whereas a $99 \%$ boundary layer is used in ref. 37 . Also the exponent $\alpha_{\mathrm{BL}}=$ $0.45 \pm 0.02$ lies slightly below the theoretical exponent of $1 / 2$. This is due to the finite spreading diameter of the droplet: an instantaneous boundary layer development over the full surface is assumed in the theory, ${ }^{37}$ but the actual boundary layer development is limited to the (growing) area below the droplet. The deviations from the theoretical relationship $\left(\delta \sim D_{0} \sqrt{t / \tau \mathrm{Re}}\right)$ are smaller than the differences originating from different boundary layer thickness definitions. Therefore, for practical purposes, either relationship will adequately describe the boundary layer development in droplet impact.

\section{E. Rim development}

The formation of a pronounced rim is observed both in experiments (Fig. 6) and simulations (Fig. 11). To quantify the rim diameter, a circle is fitted to the outermost points of the simulated droplet profile as plotted in Fig. 11 (dashed white circles collapsing to the rim). The resulting rim diameter is plotted as a function of time in Fig. 13a, in which the markers show validation measurements from the side-view images which confirm the numerical results.

In Fig. 13b, the rim radius is normalized by a length scale $D_{0} /$ $\mathrm{We}^{1 / 2} \cdot{ }^{64}$ This length scale is derived from the position which the rim has reached after a time $\tau=D_{0} / V_{0}$, assuming a typical deceleration of $V_{0}{ }^{2} / D_{0}{ }^{21,64}$ Using this normalization, all our data collapse to a single curve described by $D_{\text {Rim }} / D_{0} \sim \mathrm{We}^{-1 / 2}(t / \tau)^{\alpha_{\text {Rim }}}$. Fitting our data to this equation provides $\alpha_{\text {Rim }}=0.68 \pm 0.04$. To the best of our knowledge, this remarkably robust scaling exponent (which seems to hold also for times $t / \tau>1$ ) has not yet been theoretically explained.

To compare our results to rim size measurements of mmsized droplets, measurements by de Ruiter et al. ${ }^{64}$ are added to Fig. 13b (as discussed in ref. 64, earlier experimental work on the rim thickness ${ }^{27,57,65-67}$ is hardly applicable to drop impact on a smooth solid surface). These measurements neither collapse on our data nor our numerical results, but they are of the same order of magnitude. The temporal evolution of the rim diameter could not be uniquely established in ref. 64 as the data set was limited to $0.2<t / \tau<1$. However, since droplet impact is generally scale-invariant, and our results partially agree to theory and experiments for mm-sized droplets, ${ }^{64}$ the scaling observed herein might also describe the rim dynamics of $\mathrm{mm}$ sized droplets.

\section{F. Maximum spreading diameter}

The measured maximum spreading diameter $D_{\max } / D_{0}$ is plotted in Fig. 14 and compared to several theoretical ${ }^{21,26,37-44}$ and experimental results for microdroplets. ${ }^{3,14,15,20,31}$ For We $\approx 100$, good agreement is observed with previous experiments. ${ }^{14,31}$ For larger Weber numbers, the current maximum diameter clearly lies below ${ }^{15,20}$ or above ${ }^{3,14,31}$ previous work; the differences are discussed in the Appendix.

The maximum spreading diameter is the result of a competition of forces. On the one hand, inertia drives the liquid to radially expand. On the other hand, surface tension pulls back the expanding sheet, and viscosity dissipates part of the 


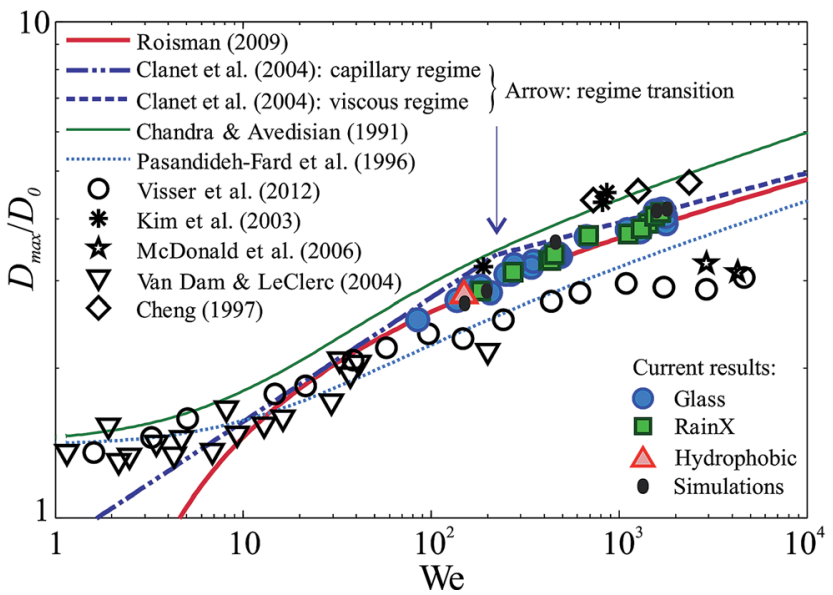

Fig. 14 Normalized maximum spreading diameter versus the Weber number, for the current experiments (colored markers) and simulations (black dots), other micro-droplet experiments (black markers), and models (lines). The arrow indicates the transition between the capillary- and viscous-regimes proposed in ref. 21.

energy, limiting the maximum spreading diameter. The limiting cases, in which inertia is balanced by either surface tension or viscous dissipation (hereafter called the capillaryand viscous regime, respectively), were separately modeled by Clanet et al. ${ }^{\mathbf{2 1}}$ The transition between these regimes is set by the parameter $P=\mathrm{We} / \mathrm{Re}^{4 / 5}=1$, as indicated by the arrow in Fig. 14 and the solid line in Fig. 2. The model shows good agreement with the current results.

However, two important additional phenomena affect the maximum spreading diameter: ${ }^{26,36,37,54,68}$ (i) the formation of a rim at the edge of the expanding droplet and (ii) viscous dissipation takes place within a thin boundary layer, as discussed. An improved approach to model spreading at We $\gg 1$ would therefore include inertial spreading limited by boundary layer dissipation and the retraction of a rim. This approach was taken by Roisman, ${ }^{37}$ where the scaling of $D_{\max } /$ $D_{0} \sim \mathrm{Re}^{1 / 5}$ for viscosity-limited spreading is recovered analogous to ref. 21 (for Re $\gtrsim 100$ and infinite Weber number). This result was generalized for finite Weber numbers (We $\gtrsim 10$ ) by introducing a correction to account for the growth of the rim, resulting in the following semi-empirical expression for the maximal spreading diameter: ${ }^{37}$

$$
D_{\max } / D_{0}=0.87 \mathrm{Re}^{1 / 5}-0.40 \mathrm{Re}^{2 / 5} \mathrm{We}^{-1 / 2}
$$

Excellent agreement with our data is observed (Fig. 14). As this model was also validated for mm-sized droplets, we conclude that, for a large parameter regime, the maximum spreading diameter is captured by the Weber- and Reynolds numbers alone.§

$\S$ As discussed in ref. 26, excluding the boundary layer generally results in an over-prediction of $D_{\max }$. In this view, the excellent agreement between our measurements and the basic model from ref. 21 is surprising. However, as the spreading stops when the boundary layer equals the lamella thickness, assuming the lamella thickness as the relevant viscous length scale (instead of explicitly incorporating the boundary layer) results in a very minor error.

\section{G. Splashing}

Splashing is not observed in the current and previous ${ }^{31}$ measurements for high-speed impact of microdroplets. Empirical splash threshold models sometimes (correctly) predict the absence of a splash, ${ }^{69-71}$ but also the incidence of a splash is frequently predicted for our maximum-velocity impacts, ${ }^{72-74}$ for which $V_{0}=50 \mathrm{~m} \mathrm{~s}^{-1}, D_{0}=50 \mu \mathrm{m}, \mathrm{Re} \approx 2500$, and $\mathrm{We} \approx 1770$, surface roughness $R_{\mathrm{a}}=5 \mathrm{~nm}$, and water and air at atmospheric pressure are used.

These conflicting results clearly indicate a need for improving the physical understanding of splashing, on which great progress has recently been made. The consensus is that splashing is caused by ejection and upward deflection of the liquid lamella formed during impact. ${ }^{56,75}$ In particular, two splash mechanisms have been proposed. First, the lamella may be deflected upwards by liquid touchdown on the substrate. This touchdown results in a local boundary layer, which has a "bump"-shape deflecting the ejected lamella away from the surface. ${ }^{75}$ The impact velocity threshold beyond which splashing occurs is proposed to equal the critical impact velocity for lamella ejection, provided as: ${ }^{75}$

$$
V_{\text {th }}=C\left(\frac{2 \sigma^{3}}{\mu_{\mathrm{g}}{ }^{2} \rho D_{0}}\right)^{1 / 4}, \quad 0.1<C<0.3
$$

providing $8.9<V_{\mathrm{th}}<27 \mathrm{~m} \mathrm{~s}^{-1}$ for our system, i.e. the predicted threshold velocity is too low. However, surface roughness and air compressibility may influence the actual threshold. ${ }^{75}$ Extrapolating Fig. 8 from ref. 75 indicates that roughness on a $1 \mathrm{~nm}$ scale (which is of the order of our glass substrate roughness) is expected to further decrease the splash threshold impact velocity. Another factor which potentially suppresses splashing for the current small-scale droplets is that the touchdown occurs in a different way as compared to the model in ref. 75 , since the air film thickness is expected to become thinner than $\sim 10$ times the mean free path length of the gas molecules. In this regime the droplet's touchdown is highly nontrivial. Although the subtleties for this case have been discussed in detail for mm-sized droplets, ${ }^{75}$ they may be different for the current regime. Extending this model to fast microdroplet impact would provide an opportunity for further validation.

A second proposed splash threshold is based on a competition between destabilization of the lamella by the air film under the droplet, and the stabilizing effect of surface tension. ${ }^{32}$ This model (which is based on dimensional analysis, and therefore does not explicitly include any physical mechanisms) predicts splashing for

$$
V_{0} \geq \frac{\rho \sigma^{2} k_{\mathrm{B}} T}{\gamma \mu M_{\mathrm{G}} P^{2} D_{0}}
$$

with $k_{\mathrm{B}}$ Boltzmann's constant, $T$ the temperature, $M_{\mathrm{G}}$ the molecular weight of the gas, $\gamma$ the adiabatic constant of the gas, and $P$ the pressure of the ambient air. For our $50 \mu \mathrm{m}$ droplets in air, this yields a splashing threshold velocity of $\sim 500 \mathrm{~m} \mathrm{~s}^{-1}$, thus no splashing is predicted for our droplets with impact velocities up to $50 \mathrm{~m} \mathrm{~s}^{-1}$. 
Recently, a similar approach has been translated into a complete physical model. Here, the lift-off of the lamella is assumed to be governed by the air surrounding the droplet (by both air compression below the lamella and a lift force due to a low-pressure area above the lamella). ${ }^{56}$ Another key ingredient is the (growing) rim diameter. If the lamella is lifted faster than the rim grows, the rim will not touch the substrate and splashing is predicted. However, if the rim diameter increases faster than the lamella lifts away from the surface, the rim will touch the substrate and splashing is suppressed. This model successfully predicts the splash threshold of mm-sized droplets, for a variety of liquids. However, a key model assumption is that the air film thickness exceeds the mean free path length of the gas molecules. As this condition is violated here, a prediction cannot be obtained for our control parameters.

As yet, our observation of non-splashing impact is predicted only by empirical models..$^{32,69,70}$ As the model by $\mathrm{Xu}$ et al. ${ }^{32}$ contains most physical insights and has been successfully extended to explain the splash threshold of droplets impacting on hot surfaces, ${ }^{29}$ it seems to be the best splash prediction model as yet. However, since only scaling arguments are used, details of the physics of splashing remain to be understood. Recent attempts provide validated splash threshold predictions for mm-sized droplets, ${ }^{56,75}$ but neither of these approaches is applicable to the micrometer domain since the continuum assumption of the gas breaks down. As the underlying physics of these models are fundamentally different, details of the splash mechanism remain to be fully understood and future modeling efforts are expected. The current results, in which no splash is observed despite high impact velocities, may provide a powerful tool for validating these models.

\section{Conclusions}

In this work the similarities and differences between mm-sized droplet impact (for which great progress has been made recently) and micrometer-sized droplet impact (which is key for industrial applications but has hardly been addressed) are studied in detail. Highly reproducible micrometer-scale droplets were generated and visualized using flash-illumination. Side-view images show the spreading droplet at a temporal resolution exceeding $10^{7}$ frames per second. Bottom-view images reveal a fringe pattern due to reflection of the (flat) glass interface and the (curved) top surface of the droplet, and were used to resolve the droplet shape at sub-micron resolution. A numerical model is developed and extensively validated using the side- and bottom-view measurements. The model is used to study the boundary layer development and the size of the rim, which are challenging to quantify experimentally.

The spreading dynamics, droplet-air interaction, boundary layer development, rim development, maximum droplet spreading diameter, and splashing threshold are investigated in detail. The main conclusions are:

1. The spreading dynamics can be divided into different phases. The initial phase, prior to the ejection of a lamella, could not be investigated due to the extremely short time scales involved. In the lamella phase, a scaling $D / D_{0} \sim \sqrt{t / \tau}$ is observed, which is in quantitative agreement with ref. 57. The final (slow-down) phase results in the maximal spreading diameter, see item 5 .

2. The air bubble size entrained during microdroplet impact decreases with increasing impact velocities (for the currently studied impact velocities $V_{0}>10 \mathrm{~m} \mathrm{~s}^{-1}$ ). The scaling of the air bubble size collapses to results for $\mathrm{mm}$-sized droplet impact in the high-Stokes number regime. The threshold Stokes number separating the high- and low Stokes number regimes is different, which is expected since this parameter is sizedependent.

3. The boundary layer thickness scales as $\bar{\delta}_{\mathrm{BL}}(t) \sim D_{0} \mathrm{Re}^{-1 / 2}$ $(t / \tau)^{\alpha}$ with an effective exponent $\alpha_{\mathrm{BL}}=0.45 \pm 0.02$. The Reynolds number dependence follows the expected scaling of $\bar{\delta}_{\mathrm{BL}}(t) \sim D_{0} \mathrm{Re}^{-1 / 2} \sqrt{t / \tau}$, but the temporal progression is somewhat lower. In addition, details of the definition of the boundary layer have a pronounced effect, which is expected to influence variables which depend on the boundary-layer thickness.

4. The rim diameter follows a scaling $D_{\text {Rim }} / D_{0} \sim \mathrm{We}^{-1 / 2}(t / \tau)^{\alpha_{\text {Rim }}}$, with effective exponent $\alpha_{\text {Rim }}=0.68 \pm 0.04$. To the best of our knowledge, this scaling is observed for the first time. The temporal exponent and the remarkable robustness of this scaling for times $t / \tau>1$ remain to be explained.

5. The maximal droplet spreading diameter is robustly captured by models balancing the inertia of the droplet to viscous dissipation in the boundary layer. ${ }^{37}$

6. Despite our high impact velocities up to $V_{0}=50 \mathrm{~m} \mathrm{~s}^{-1}$, no splash is observed here. Only the model by Xu et al. ${ }^{32}$ correctly predicts this outcome. This model is based on scaling arguments, and does not contain detailed (force) balances and prefactors. Alternative models capture the physics of splashing in more detail,,$^{56,75}$ but since the continuum assumption is violated for the thin air film between the droplet and the substrate these models cannot be applied here.

As far as could be observed, droplet impact is scale-invariant, i.e. $\mathrm{mm}$-sized droplet experiments have a predictive value as long as the same Reynolds, Weber, and Stokes numbers are used. Therefore, the vast progress in understanding mm-sized droplet impact can be applied to optimize a wide range of applications in which fast, $\mu$ m-sized droplet impact on a dry surface is a key process step, such as (3D) (bio)printing, thermal spraying, spray cleaning, and laser-induced forward transfer. Future studies may address additional phenomena including lamella break-up, ${ }^{76}$ the influence of the slip length, ${ }^{54,76}$ and droplet compressibility ${ }^{76,77}$ as these are highly relevant for impact of even faster and smaller droplets. ${ }^{76}$

\section{Appendix}

\section{A. Explanation of differences among experimental results of droplet spreading}

Fig. 14 shows the normalized maximum spreading diameter. For We $>100$, the current maximum diameter clearly lies below ${ }^{15,20}$ or above $^{\mathbf{3 , 1 4 , 3 1}}$ previous work; the discrepancies are discussed here.

The results exceeding our results were taken for droplets with $D_{0}=235 \pm 30 \mu \mathrm{m}$ (ref. 15) and $D_{0}=230 \mu \mathrm{m},{ }^{20}$ i.e. larger 
than our droplets with $D_{0}=48 \pm 3 \mu \mathrm{m}$. As the spreading depends on both We and Re (and not on We alone as displayed in the graph), a larger spreading is expected. Assuming We $=$ 800 and droplet diameters $D_{0}=[48,235] \mu \mathrm{m}$, we obtain $\mathrm{Re}=$ [1661, 3666], respectively. Using the model of Roisman, ${ }^{37}$ a $15 \%$ larger spreading diameter is expected for the larger $\left(D_{0}=230\right.$ $\mu \mathrm{m})$ droplets. The data around $\mathrm{We}=800$ shows a $20 \%$ increase, i.e. a slightly larger but similar value.

Our previous results ${ }^{31}$ are clearly lower than the current results (at $\mathrm{We}=800$, a $21 \%$ decrease is found). This is due to three reasons. First, the high-Weber number measurements were taken for smaller droplets $(\sim 20 \mu \mathrm{m})$, as the previous droplet generation method did not produce consistent droplet sizes for all velocities (ref. 31, Fig. 2c). An analysis similar to the previous paragraphs shows an expected decrease in spreading diameter of $5 \%$. Second, the current measurements show that the maximum spreading is reached by the cylindrical rim, i.e. above the surface. Just after reaching its maximum spreading the rim retracts around $3 \%$, while (for hydrophilic surfaces) the contact line initially sticks to the surface. The temporal resolution of the previous measurements $(1 \mu \mathrm{s})$ did not allow the visualization of this retraction, and thus in the previous measurement the maximum wetted diameter was measured instead of the maximum extension of the rim. This also accounts for a part of the discrepancy. Finally, the spatial resolution of the previous experiments was much more limited (ref. 31, Fig. 3d). In that work we appear to have over-estimated the measurement accuracy at the droplet edge, and we now conclude that high-resolution measurements are required for adequate spreading measurements. As this problem worsens with increasing velocities, the plateau of $D_{\max } / D_{0}$ reported for We $>1000$ (ref. 20 and 31) requires validation.

Then, the maximum spreading of hot metal droplets onto a glass surface (ref. 3, Fig. 3b) also lies below current results. As the glass temperature was much below the metal melting temperature, solidification might have limited the spreading diameter. In addition, the surface tension of a hot ( $3300 \mathrm{~K})$ droplet while cooling down and oxidizing is hard to establish, i.e. the Weber number is poorly defined. Here we take a value of $\sigma=2.3 \mathrm{~N} \mathrm{~m}^{-1}$ from ref. 78 and estimate $\sigma=2.0 \mathrm{~N} \mathrm{~m}^{-1}$ for the steel-chromium alloy reported, ${ }^{79}$ as values for the actual alloy may not exist. Finally, the initial droplet diameter had a high uncertainty (particles with $D_{0}=60 \pm 38 \mu \mathrm{m}$ with an average of $D_{0}=40 \mu \mathrm{m}$ were reported). Although a 40-measurement average was taken, this could strongly add to the error. Therefore, in view of these major uncertainties, we are surprised by the reasonable agreement with our measurements.

Finally, one data point from previous high-quality measurements ${ }^{\mathbf{1 4}}$ lies significantly below our data. As this single data point also lies below the curve plotted in ref. 14, we took a detailed look at the experimental parameters. The authors report a maximum impact velocity of $V_{0}=13.8 \mathrm{~m} \mathrm{~s}^{-1}$ and a maximum diameter of $D_{0}=42 \mu \mathrm{m}$, corresponding to $\mathrm{We}=111$ for water. However, the data point was plotted at $\mathrm{We} \approx 200$, resulting in a minor discrepancy. In conclusion, we think the current results are at least as reliable as (and mostly consistent with) previous work.

\section{Acknowledgements}

We thank H. Gelderblom, A. Lippert and W. Bouwhuis for stimulating discussions, and M. N. Hultmark for providing superhydrophobic samples. The financial support by the Austrian Federal Ministry of Economy, Family and Youth, the Austrian National Foundation for Research, Technology and Development, and the Foundation for Fundamental Research of Matter (FOM) is gratefully acknowledged. The authors would like to thank Lam Research AG for support.

\section{References}

1 S. W. Park and C. S. Lee, Macroscopic and microscopic characteristics of a fuel spray impinged on the wall, Exp. Fluids, 2004, 37, 745.

2 A. Moreira, A. Moita and M. Panão, Advances and challenges in explaining fuel spray impingement: How much of single droplet impact research is useful?, Prog. Energy Combust. Sci., 2010, 36, 554.

3 A. McDonald, M. Lamontagne, C. Moreau and S. Chandra, Impact of plasma-sprayed metal particles on hot and cold glass surfaces, Thin Solid Films, 2006, 514, 212.

4 H. Troesch, PhD Thesis, Die Zerstäubung von Flüssigkeiten, Federal Technology College in Zurich, 1954.

5 D. Attinger, Z. Zhao and D. Poulikakos, An experimental study of molten microdroplet surface deposition and solidification: Transient behavior and wetting angle dynamics, J. Heat Transfer, 2000, 122, 544.

6 S. Tasoglu and U. Demirci, Bioprinting for stem cell research, Trends Biotechnol., 2013, 31, 10.

7 T. Boland, X. Tao, B. J. Damon, B. Manley, P. Kesari, S. Jalota and S. Bhaduri, Drop-on-demand printing of cells and materials for designer tissue constructs, Mater. Sci. Eng., C, 2007, 27, 372 .

8 B. Guillotin, A. Souquet, S. Catros, M. Duocastella, B. Pippenger, S. Bellance, R. Bareille, M. Rémy, L. Bordenave, J. Amédée and F. Guillemot, Laser assisted bioprinting of engineered tissue with high cell density and microscale organization, Biomaterials, 2010, 31, 7250.

9 A. Klini, P. A. Loukakos, D. Gray, A. Manousaki and C. Fotakis, Laser induced forward transfer of metals by temporally shaped femtosecond laser pulses, Opt. Express, 2008, 16, 11300.

10 J. Schlabe, C. Johnen, R. Schwartlander, V. Moser, B. Hartmann, J. C. Gerlach and M. V. Küntscher, Isolation and culture of different epidermal and dermal cell types from human scalp suitable for the development of a therapeutical cell spray, Burns, 2008, 34, 376.

11 H. F. Okorn-Schmidt, F. Holsteyns, A. Lippert, D. Mui, M. Kawaguchi, C. Lechner, P. E. Frommhold, T. Nowak, F. Reuter, M. B. Pique, C. Cairos and R. Mettin, Particle cleaning technologies to meet advanced semiconductor device process requirements, ECS J. Solid State Sci. Technol., 2013, 3, N3069.

12 W. Melfo, H. Bolt, M. Rijnders, D. Staalman, C. B. Castro, D. Crowther and B. Jana, Experimental study on primary 
scale formation and descalability on steels containing $\mathrm{Ni}$ and $\mathrm{Ni}+\mathrm{Si}$, ISIJ Int., 2013, 53, 866.

13 M. Versluis, High-speed imaging in fluids, Exp. Fluids, 2013, 54, 1458.

14 D. B. van Dam and C. Le Clerc, Experimental study of the impact of an ink-jet printed droplet on a solid substrate, Phys. Fluids, 2004, 16, 3403.

15 H.-Y. Kim, S.-Y. Park and K. Min, Imaging the high-speed impact of microdrop on solid surface, Rev. Sci. Instrum., 2003, 74, 4930.

16 S. Jung and I. M. Hutchings, The impact and spreading of a small liquid drop on a non-porous substrate over an extended time scale, Soft Matter, 2012, 8, 2686.

17 R. C. A. van der Veen, T. Tran, D. Lohse and C. Sun, Direct measurements of air layer profiles under impacting droplets using high-speed color interferometry, Phys. Rev. E: Stat., Nonlinear, Soft Matter Phys., 2012, 85, 026315.

18 R. Bhola and S. Chandra, Parameters controlling solidification of molten wax droplets falling on a solid surface, J. Mater. Sci., 1999, 4, 4883.

19 W. Bouwhuis, R. C. A. van der Veen, T. Tran, D. L. Keij, K. G. Winkels, I. R. Peters, D. van der Meer, C. Sun, J. H. Snoeijer and D. Lohse, Maximal air bubble entrainment at liquid-drop impact, Phys. Rev. Lett., 2012, 109, 264501.

20 L. Cheng, Dynamic spreading of drops impacting onto a solid surface, Ind. Eng. Chem. Process Des. Dev., 1977, 16, 192.

21 C. Clanet, C. Béguin, D. Richard and D. Quéré, Maximal deformation of an impacting drop, J. Fluid Mech., 2004, 517, 199.

22 R. Ford and C. Furmidge, Impact and spreading of spray drops on foliar surfaces, Soc. Chem. Ind. Monogr., 1967, 417.

$23 \mathrm{H}$. Fukanuma and A. Ohmori, in Proceedings of the 7th National Thermal Spray Conference, ASM International, Boston, MA, 1994, pp. 563-568.

24 G. Lagubeau, M. A. Fontelos, C. Josserand, A. Maurel, V. Pagneux and P. Petitjeans, Spreading dynamics of drop impacts, J. Fluid Mech., 2012, 713, 50.

$25 \mathrm{H}$. Marmanis and S. T. Thoroddsen, Scaling of the fingering pattern of an impacting drop, Phys. Fluids, 1996, 8, 1344.

26 M. Pasandideh-Fard, Y. M. Qiao, S. Chandra and J. Mostaghimi, Capillary effects during droplet impact on a solid surface, Phys. Fluids, 1996, 8, 650.

27 C. D. Stow and M. G. Hadfield, An experimental investigation of fluid flow resulting from the impact of a water drop with an unyielding dry surface, Proc. R. Soc. A, 1981, 373, 419.

28 S. Thoroddsen and J. Sakakibara, Evolution of the fingering pattern of an impacting drop, Phys. Fluids, 1998, 10, 1359.

29 T. Tran, H. J. J. Staat, A. Prosperetti, C. Sun and D. Lohse, Drop impact on superheated surfaces, Phys. Rev. Lett., 2012, 108, 036101.

30 P. Tsai, R. C. A. van der Veen, M. van de Raa and D. Lohse, How micropatterns and air pressure affect splashing on surfaces, Langmuir, 2010, 26, 16090.

31 C. W. Visser, Y. Tagawa, C. Sun and D. Lohse, Microdroplet impact at very high velocity, Soft Matter, 2012, 8, 10732.
32 L. Xu, W. Zhang and S. Nagel, Drop splashing on a dry smooth surface, Phys. Rev. Lett., 2005, 94, 184505.

33 L. Xu, L. Barcos and S. Nagel, Splashing of liquids: Interplay of surface roughness with surrounding gas, Phys. Rev. E: Stat., Nonlinear, Soft Matter Phys., 2007, 76, 066311.

34 M. Rein, Phenomena of liquid drop impact on solid and liquid surfaces, Fluid Dyn. Res., 1993, 12, 61.

35 A. Yarin, Drop impact dynamics: Splashing, spreading, receding, bouncing, Annu. Rev. Fluid Mech., 2006, 38, 159.

36 J. Eggers, M. A. Fontelos, C. Josserand and S. Zaleski, Drop dynamics after impact on a solid wall: Theory and simulations, Phys. Fluids, 2010, 22, 062101.

37 I. V. Roisman, Inertia dominated drop collisions. II. An analytical solution of the Navier-Stokes equations for a spreading viscous film, Phys. Fluids, 2009, 21, 052104.

38 J. M. Kolinski, S. M. Rubinstein, S. Mandre, M. P. Brenner, D. A. Weitz and L. Mahadevan, Skating on a film of air: Drops impacting on a surface, Phys. Rev. Lett., 2012, 108, 074503.

39 M. M. Driscoll and S. R. Nagel, Ultrafast interference imaging of air in splashing dynamics, Phys. Rev. Lett., 2011, 107, 154502.

40 S. Mandre, M. Mani and M. P. Brenner, Precursors to splashing of liquid droplets on a solid surface, Phys. Rev. Lett., 2009, 102, 134502.

41 M. Mani, S. Mandre and M. Brenner, Events before droplet splashing on a solid surface, J. Fluid Mech., 2010, 647, 163.

42 S. T. Thoroddsen, T. G. Etoh, K. Takehara, N. Ootsuka and Y. Hatsuki, The air bubble entrapped under a drop impacting on a solid surface, J. Fluid Mech., 2005, 545, 203.

43 J. S. Lee, B. M. Weon, J. H. Je and K. Fezzaa, How does an air film evolve into a bubble during drop impact?, Phys. Rev. Lett., 2012, 109, 204501.

44 S. Chandra and C. T. Avedisian, On the dollision of a droplet with a solid surface, Proc. R. Soc. A, 1991, 432, 13.

45 V. Mehdi-Nejad, J. Mostaghimi and S. Chandra, Air bubble entrapment under an impacting droplet, Phys. Fluids, 2003, 15, 173.

46 S. D. Aziz and S. Chandra, Impact, recoil and splashing of molten metal droplets, Int. J. Heat Mass Transfer, 2000, 43, 2841.

47 D. Richard, C. Clanet and D. Quéré, Contact time of a bouncing drop, Nature, 2002, 417, 811.

48 M. Reyssat, D. Richard, C. Clanet and D. Quéré, Dynamical superhydrophobicity, Faraday Discuss., 2010, 146, 19.

49 A. Checco, P. Guenoun and J. Daillant, Nonlinear Dependence of the Contact Angle of Nanodroplets on Contact Line Curvature, Phys. Rev. Lett., 2003, 91, 186101.

50 N. R. Lindblad and J. M. Schneider, Production of uniformsized liquid droplets, J. Sci. Instrum., 1965, 42, 635.

51 P. E. Frommhold, A. Lippert, F. L. Holsteyns and R. Mettin, High-speed monodisperse droplet generation by ultrasonically controlled micro-jet breakup, Exp. Fluids, 2014, 55, 1716. 
52 P. Kim, M. J. Kreder, J. Alvarenga and J. Aizenberg, Hierarchical or not? Effect of the length scale and hierarchy of the surface roughness on omniphobicity of lubricant-infused substrates, Nano Lett., 2013, 13, 1793.

53 S. Popinet, An accurate adaptive solver for surface-tensiondriven interfacial flows, J. Comput. Phys., 2009, 228, 5838.

54 H. Lastakowski, F. Boyer, A. L. Biance, C. Pirat and C. Ybert, Bridging local to global dynamics of drop impact onto solid substrates, J. Fluid Mech., 2014, 747, 103.

55 R. Rioboo, M. Marengo and C. Tropea, Time evolution of liquid drop impact onto solid, dry surfaces, Exp. Fluids, 2002, 33, 112.

56 G. Riboux and J. M. Gordillo, Experiments of drops impacting a smooth solid surface: A model of the critical impact speed for drop splashing, Phys. Rev. Lett., 2014, 113, 024507.

57 A. Mongruel, V. Daru, F. Feuillebois and S. Tabakova, Early post-impact time dynamics of viscous drops onto a solid dry surface, Phys. Fluids, 2009, 21, 032101.

58 H.-Y. Kim, Z. C. Feng and J.-H. Chun, Instability of a liquid jet emerging from a droplet upon collision with a solid surface, Phys. Fluids, 2000, 12, 531.

$59 \mathrm{H}$. Wagner, Phenomena associated with impacts and sliding on liquid surfaces, Z. Angew. Math. Mech., 1932, 4, 1-61.

60 P. D. Hicks and R. Purvis, Air cushioning in droplet impacts with liquid layers and other droplets, Phys. Fluids, 2011, 23, 062104.

61 J. M. Kolinski, L. Mahadevan and S. M. Rubinstein, Lift-off instability during the impact of a drop on a solid surface, Phys. Rev. Lett., 2014, 112, 134501.

62 A. Eddi, K. G. Winkels and J. H. Snoeijer, Short time dynamics of viscous drop spreading, Phys. Fluids, 2013, 25, 013102.

63 P. D. Hicks and R. Purvis, Liquid-solid impacts with compressible gas cushioning, J. Fluid Mech., 2013, 735, 120.

64 J. de Ruiter, R. E. Pepper and H. A. Stone, Thickness of the rim of an expanding lamella near the splash threshold, Phys. Fluids, 2010, 22, 022104.

65 D. C. D. Roux and J. J. Cooper-White, Dynamics of water spreading on a glass surface, J. Colloid Interface Sci., 2004, 277, 424.
66 I. V. Roisman, K. Horvat and C. Tropea, Spray impact: Rim transverse instability initiating fingering and splash, and description of a secondary spray, Phys. Fluids, 2006, 18, 102104.

67 E. Villermaux and B. Bossa, Drop fragmentation on impact, J. Fluid Mech., 2011, 668, 412.

68 S. Tabakova, F. Feuillebois, A. Mongruel, V. Daru and S. Radev, First stages of drop impact on a dry surface: Asymptotic model, Z. Angew. Math. Phys., 2012, 63, 313.

69 R. L. V. Wal, G. M. Berger and S. D. Mozes, The splash/nonsplash boundary upon a dry surface and thin fluid film, Exp. Fluids, 2005, 40, 53.

70 C. S. Stevens, Scaling of the splash threshold for lowviscosity fluids, Europhys. Lett., 2014, 106, 24001.

71 K.-L. Pan, K.-C. Tseng and C.-H. Wang, Breakup of a droplet at high velocity impacting a solid surface, Exp. Fluids, 2009, 48, 143.

72 C. Mundo, M. Sommerfeld and C. Tropea, Droplet-wall collisions: Experimental studies of the deformation and breakup process, Int. J. Multiphase Flow, 1995, 21, 151.

$73 \mathrm{~K}$. Range and F. Feuillebois, Influence of surface roughness on liquid drop impact, J. Colloid Interface Sci., 1998, 30, 16.

74 G. E. Cossali, A. Coghe and M. Marengo, The impact of a single drop on a wetted solid surface, Exp. Fluids, 1997, 22, 463.

75 S. Mandre and M. Brenner, The mechanism of a splash on a dry solid surface, J. Fluid Mech., 2012, 690, 148.

76 R. Zhang, S. Farokhirad, T. Lee and J. Koplik, Multiscale liquid drop impact on wettable and textured surfaces, Phys. Fluids, 2014, 26, 082003.

77 J. Field, J. Dear and J. Ogren, The effects of target compliance on liquid drop impact, J. Appl. Phys., 1989, 65, 533.

78 W. M. Haynes, D. R. Lide, and T. J. Bruno, CRC Handbook of Chemistry and Physics, CRC Press, Boca Raton, Fl, USA, 2012.

79 K. Mukai, L. Zushu and M. Zeze, Surface tension and wettability of liquid Fe-16 mass\% Cr-O alloy with alumina, Mater. Trans., 2002, 43, 1724. 